\title{
Nonnegative solutions to an elliptic problem with nonlinear absorption and a nonlinear incoming flux on the boundary
}

\author{
J. García-Melián ${ }^{1}$, C. Morales-Rodrigo ${ }^{2}$, J. D. Rossi $^{3}$ and A. Suárez $^{2,4}$, \\ 1. Dpto. de Análisis Matemático, Universidad de La Laguna, \\ C/. Astrofísico Francisco Sánchez s/n, 38271 - La Laguna, SPAIN, \\ 2. Dpto. de Ecuaciones Diferenciales y Análisis Numérico \\ Fac. de Matemáticas, Univ. de Sevilla \\ C/. Tarfia s/n, 41012 - Sevilla, SPAIN,
}

3. Facultad de Ciencias Exactas y Naturales, Universidad de Buenos Aires,

1428 - Buenos Aires, ARGENTINA

Instituto de Matemáticas y Física Fundamental, CSIC,

C/. Serrano 123, 28006 - Madrid, SPAIN

E-mail addresses: jjgarmel@ull.es, cristianmatematicas@yahoo.com, jrossi@dm.uba.ar, suarez@us.es

\begin{abstract}
In this paper we perform an extensive study of the existence, uniqueness (or multiplicity) and stability of nonnegative solutions to the semilinear elliptic equation $-\Delta u=\lambda u-u^{p}$ in $\Omega$, with the nonlinear boundary condition $\partial u / \partial \nu=u^{r}$ on $\partial \Omega$. Here $\Omega$ is a smooth bounded domain of $\mathbb{R}^{d}$ with outward unit normal $\nu, \lambda$ is a real parameter and $p, r>0$. We also give the precise behavior of solutions for large $|\lambda|$ in the cases where they exist. The proofs are mainly based on bifurcation techniques, sub-supersolutions and variational methods.
\end{abstract}

Key Words. Elliptic equations, Nonlinear boundary conditions.

\section{Introduction and main results}

Consider a bounded domain $\Omega \subset \mathbb{R}^{d}, d \geq 2$, with a $C^{2, \gamma}$ boundary, $\partial \Omega, 0<\gamma<1$. We are interested in the study of positive solutions to the problem

$$
\left\{\begin{aligned}
-\Delta u & =\lambda u-u^{p} & & \text { in } \Omega, \\
\frac{\partial u}{\partial \nu} & =u^{r} & & \text { on } \partial \Omega,
\end{aligned}\right.
$$

\footnotetext{
${ }^{4}$ Corresponding author: Fax number: +34954552898
} 
where $p, r>0, \lambda \in \mathbb{R}$ will be regarded as a bifurcation parameter and $\nu$ is the outward normal vector field to $\partial \Omega$.

The study of elliptic problems with nonlinear boundary conditions has attracted a great attention in the last decade, see the survey [38] and references therein. In problem (1.1) there is a competition between the absorption term in the equation and the positive flux at the boundary. Thus, it is interesting to look how the linear term, $\lambda u$, affects the existence of positive solutions to (1.1).

Nonlinear boundary conditions appear in a rather natural way in some physical models, see [38]. In the particular case $p>1$, problem (1.1) can be given an ecological meaning, since the equation is the well-known logistic equation, which models the diffusion of a single species in the habitat $\Omega$ whose density is given by $u$. The boundary condition means that the individuals are taken outside the habitat once they reach the boundary $\partial \Omega$, at a rate which also depends on $u$ through a power, see [12] for a related problem arising from population dynamics with a different nonlinearity on the boundary condition.

Problem (1.1) has been studied previously in different papers. In [32] some particular results have been given for $p, r>1$ showing that there exists positive solution for all $\lambda \geq 0$ if $p>2 r-1$ and for $\lambda<\Lambda_{0}$ (for some $\Lambda_{0} \geq 0$ ) if $p<2 r-1$ and $r<d /(d-2$ ).

In the particular cases $\lambda=0, p, r>1$ and the nonlinearity in the equation is $-a u^{p}$ when $a \in \mathbb{R}$ varies, problem (1.1) has been analyzed in [14], [15], [29] and [35] (see also references therein). For these specific values of $\lambda, p$ and $r$, it is proved that, if $p<r$ or $p>2 r-1$ there is a positive solution of (1.1) for $a>0$. When $p=r$, there is a positive solution if $a>|\partial \Omega| /|\Omega|$, and there is no positive solution of (1.1) if $a<|\partial \Omega| /|\Omega|$ (throughout the paper $|\Omega|$ and $|\partial \Omega|$ will denote the $d$-dimensional and $(d-1)$-dimensional measures of $\Omega$ and $\partial \Omega$, respectively). If $r<p<2 r-1$ there exists $a_{0}>0$ such that there exists positive solution when $a>a_{0}$ and no positive solutions for $a<a_{0}$. Moreover, if $r<d /(d-2)$ then for a.a. $a \geq a_{0}(1.1)$ has at least two positive solutions. In fact, a more detailed analysis is made for the cases $d=1$ and $\Omega$ a ball (see also [31] for a one-dimensional analysis). This study shows that $p=2 r-1$ is critical in many aspects. In particular, for the corresponding time-dependent problem some solutions blow-up in finite time if and only if $p \leq 2 r-1$ (and $a<r$ if $p=2 r-1$ ). A detailed study is made in [37] even in the case $p, r \leq 1$. Moreover, if $p=2 r-1, a=r$ and $d=1$ then there exists a singular positive equilibrium and all positive solutions of its corresponding time-dependent problem are global and tend to this singular solution as $t \rightarrow \infty$, see [17].

Finally, the case $r=1, \lambda=0$, both for $p>1$ and $p<1$, and with a parameter in the boundary condition, is considered in [20] and [21].

On the other hand, when instead of a positive flux at the boundary, there is a negative one, the problem has been analyzed in [10] in the case $p, r>1$. Also, if a bounded function $g(u)$ appears in the boundary condition instead of $u^{r}$, it has been studied in [41], and for more general nonlinearities in [42], where a local bifurcation analysis is carried out using a Lyapunov-Schmidt reduction. We again refer to [38] for further information.

In this paper we continue the study of (1.1) when $p, r>1$, in the cases $p>2 r-1$ and $p<2 r-1$ completing and improving the results of [32]. Also we consider with detail the cases $r=1$ and $p>0 ; p=1$ and $r>0 ; 0<r<1<p$ and $0<p<1<r$. Observe that in the case $p=r=1$, the problem becomes linear, and hence a positive solution exists only for a value of $\lambda$, the principal eigenvalue; see Lemma 2.2. We remark that in most cases we are only considering a subcritical exponent, $r$, that is $r<d /(d-2)$ when $d \geq 3$. See Theorems 1.1-1.4 where we summarize the main results. The cases $p, r<1 ; p=2 r-1$ 
and a detailed study of the case $d=1$ will be analyzed elsewhere. We remark that all our results are valid also for $d=1$.

Our main goal is to determine the set of $\lambda$ 's for which solutions exist, as well as to determine the stability and uniqueness of the positive solutions, according to the values of $p$ and $r$. We also provide the precise asymptotic behavior of the solutions when $|\lambda|$ becomes large, in those cases where solutions exist.

Since we are only interested in nonnegative solutions to (1.1), we can extend the functions $\lambda u-u^{p}$ and $u^{r}$ to be zero for negative values of $u$. In this case, any solution to (1.1) is nonnegative. Moreover, when $p \geq 1$ the strong maximum principle implies that any nonnegative and nontrivial solution to (1.1) is positive. In the case $p<1$, the solutions could develop a dead core, but we are not analyzing this phenomenon in the present work (see [21] for a related situation). We also remark that weak solutions to (1.1) in $H^{1}(\Omega)$ are smooth up to the boundary (see Lemma 2.1).

Before proceeding to the statement of the theorems, we need to introduce some notation. Given $m \in L^{\infty}(\Omega), h \in C^{1}(\partial \Omega)$ we denote by $\lambda_{1}(-\Delta+m, N+h)$ the principal eigenvalue of the problem

$$
\left\{\begin{array}{cl}
-\Delta u+m(x) u=\lambda u & \text { in } \Omega \\
\frac{\partial u}{\partial \nu}+h(x) u=0 & \text { on } \partial \Omega,
\end{array}\right.
$$

(the notation $N$ refers to the Neumann boundary condition). Some important properties of this eigenvalue will be recalled in Section 2 (see Lemma 2.2). We only quote for the moment that for constant $m$ it holds $\lambda_{1}(-\Delta+m, N)=m$.

We are using the principal eigenvalues to characterize the stability of the solutions with respect to the parabolic counterpart problem. We say that a positive solution $u_{0}$ of (1.1) is stable (resp. unstable) if the principal eigenvalue of the linearization of (1.1) around $u_{0}$ is positive (resp. negative), i. e.,

$$
\lambda_{1}\left(-\Delta-\lambda+p u_{0}^{p-1}, N-r u_{0}^{r-1}\right)>0 \quad(\text { resp. }<0) .
$$

We also say that $u_{0}$ is weakly stable if the eigenvalue is nonnegative, and neutrally stable if it is zero.

We are now able to state our results.

\section{Theorem 1.1.}

1. Assume $r=1$ and $p \neq 1$. There exists a nonnegative and nontrivial solution if and only if $\lambda>\lambda_{1}(-\Delta, N-1)$. Moreover,

(a) if $p>1$, the solution is positive, unique (denoted by $u_{\lambda}$ ), stable and verifies

$$
\lim _{\lambda \backslash \lambda_{1}(-\Delta, N-1)}\left\|u_{\lambda}\right\|_{\infty}=0, \quad \lim _{\lambda \nearrow+\infty}\left\|u_{\lambda}\right\|_{\infty}=+\infty
$$

(b) if $p<1$, we have for every family of nonnegative solutions $\left\{u_{\lambda}\right\}$ that

$$
\lim _{\lambda \backslash \lambda_{1}(-\Delta, N-1)}\left\|u_{\lambda}\right\|_{\infty}=+\infty, \quad \lim _{\lambda \nearrow+\infty}\left\|u_{\lambda}\right\|_{\infty}=0 .
$$

2. Assume $p=1$. 
(a) If $1<r<d /(d-2)$, there exists a positive solution if and only if $\lambda<\lambda_{1}(-\Delta+$ $1, N)$. Moreover, all solutions are unstable and for every family of positive solutions $\left\{u_{\lambda}\right\}$ it holds

$$
\lim _{\lambda / \lambda_{1}(-\Delta+1, N)}\left\|u_{\lambda}\right\|_{\infty}=0, \quad \lim _{\lambda \searrow-\infty}\left\|u_{\lambda}\right\|_{\infty}=+\infty .
$$

(b) If $r<1$, there exists a positive solution if and only if $\lambda<\lambda_{1}(-\Delta+1, N)$. Moreover, the solution is unique (denoted by $u_{\lambda}$ ), stable and

$$
\lim _{\lambda / \lambda_{1}(-\Delta+1, N)}\left\|u_{\lambda}\right\|_{\infty}=\infty, \quad \lim _{\lambda \searrow-\infty}\left\|u_{\lambda}\right\|_{\infty}=0 .
$$

Theorem 1.2. Assume $0<r<1<p$. There exists a positive solution for all $\lambda \in \mathbb{R}$. Moreover, the solution is unique (denoted by $u_{\lambda}$ ), stable and

$$
\lim _{\lambda \searrow-\infty}\left\|u_{\lambda}\right\|_{\infty}=0, \quad \quad \lim _{\lambda \nearrow+\infty}\left\|u_{\lambda}\right\|_{\infty}=+\infty
$$

Theorem 1.3. Assume $0<p<1<r<d /(d-2)$. There exists a nonnegative and nontrivial solution for all $\lambda \in \mathbb{R}$. Moreover, for every family of positive solutions $\left\{u_{\lambda}\right\}$ :

$$
\lim _{\lambda \searrow-\infty}\left\|u_{\lambda}\right\|_{\infty}=+\infty, \quad \lim _{\lambda \nearrow+\infty}\left\|u_{\lambda}\right\|_{\infty}=0 .
$$

Theorem 1.4. Assume $p, r>1$.

1. If $p>2 r-1$, there exists $\lambda_{0}<0$ such that (1.1) has a positive solution if, and only if, $\lambda \geq \lambda_{0}$. Moreover, for every family of positive solutions $\left\{u_{\lambda}\right\}$ it holds

$$
\lim _{\lambda \nearrow+\infty}\left\|u_{\lambda}\right\|_{\infty}=+\infty .
$$

2. If $p<2 r-1$ and $r<d /(d-2)$, there exists $\Lambda_{0} \geq 0$ such that (1.1) has a positive solution if $\lambda<\Lambda_{0}$, and no positive solutions for $\lambda>\Lambda_{0}$. Moreover, if $\Lambda_{0}>0$, there exist at least two positive solutions for $\lambda \in\left(0, \Lambda_{0}\right)$ and at least a positive solution for $\lambda=\Lambda_{0}$. In addition, for every family of positive solutions $\left\{u_{\lambda}\right\}$ we have

$$
\lim _{\lambda \searrow-\infty}\left\|u_{\lambda}\right\|_{\infty}=+\infty
$$

3. If $p<r$ or $p=r$ and $|\Omega|>|\partial \Omega|$, and $r<d /(d-2)$ then $\Lambda_{0}>0$. Moreover, for every $\lambda \in\left(0, \Lambda_{0}\right)$ there exists a unique positive stable solution to (1.1).

In Figure 1 we have represented the bifurcation diagrams in all the cases. We remark that, in cases b), c), f) and h) the solutions need not be unique in spite of the drawings.

It is also important to stress that the asymptotic behavior of the solutions when $\lambda \nearrow+\infty$ or $\lambda \searrow-\infty$ in (1.2) through (1.9) is a consequence of a more precise information obtained for the solutions. Concretely, we prove that whenever positive solutions exist for large $|\lambda|$, we have estimates of the form

$$
C_{1}|\lambda|^{\theta} \leq \max u \leq C_{2}|\lambda|^{\theta}
$$




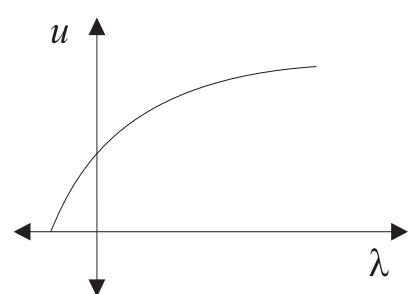

a)

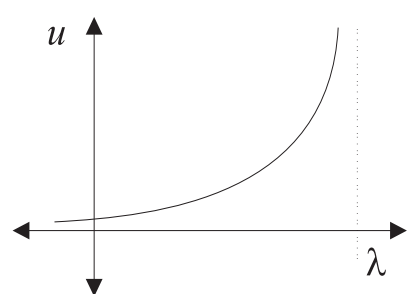

d)

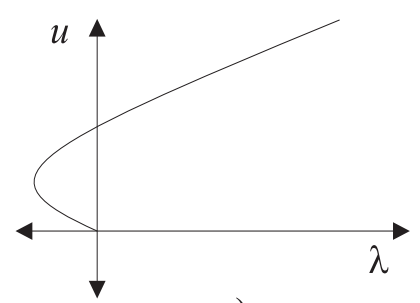

g)

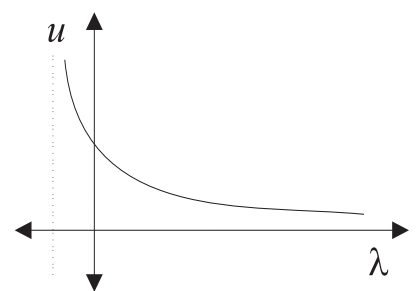

b)

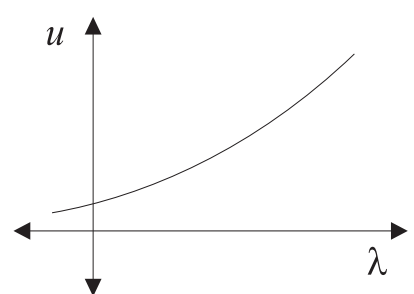

e)

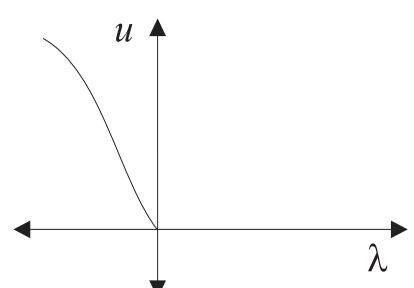

h)

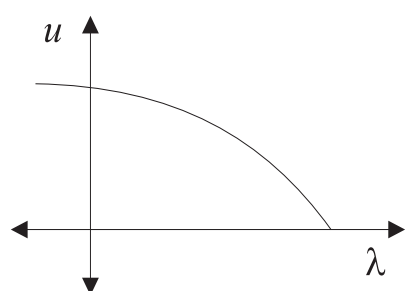

c)

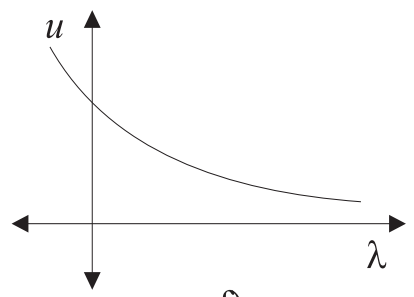

f)

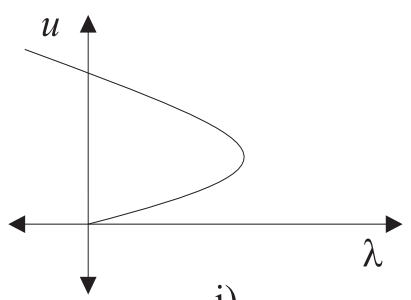

i)

Figure 1: Bifurcation diagrams of (1.1): Case a) $r=1<p$; Case b) $r=1>p$; Case c) $p=1<r<d /(d-2)$; Case d) $p=1>r$; Case e) $0<r<1<p$; Case $\mathrm{f}$ ) $0<p<1<r<d /(d-2)$; Case g) $p, r>1, p>2 r-1$; Case h) $p, r>1, p<2 r-1$, $r<d /(d-2), \Lambda_{0}=0$; Case i) $p, r>1, p<2 r-1, r<d /(d-2), \Lambda_{0}>0$. 
for every positive solution to (1.1), where $C_{1}$ and $C_{2}$ are positive constants, and the exponent $\theta$ is precisely determined in terms of $p$ and $r$. See Section 5 for the statement and proof of these results.

The paper is organized as follows: in Section 2, we collect some preliminaries needed to prove the theorems. Section 3 deals with bifurcations from infinity and from the trivial solution, while Section 4 is devoted to the proof of the existence, nonexistence and multiplicity issues. Finally, in Section 5 we analyze the precise asymptotic behavior for large $|\lambda|$.

\section{Preliminaries}

Since it will be necessary when using variational arguments, we begin this section by recalling that positive weak solutions to (1.1) are indeed classical. By a positive weak solution to (1.1) we mean a positive function $u \in H^{1}(\Omega)$ such that

$$
\int_{\Omega} \nabla u \cdot \nabla \varphi-\int_{\partial \Omega} u^{r} \varphi=\int_{\Omega}\left(\lambda u-u^{p}\right) \varphi
$$

for every $\varphi \in H^{1}(\Omega)$.

This fact is a consequence of the general regularity theory for elliptic equations (we refer to [1], [24] and [28]; see also [13] for the $C^{\infty}$ setting). Observe that in all our results when $r<1$ we are assuming that $p \geq 1$, and so by the strong maximum principle any nonnegative and nontrivial solution of (1.1) is positive.

Lemma 2.1. Let $u \in H^{1}(\Omega)$ be a nonnegative weak solution to (1.1), where $p>0$ and $0<r<d /(d-2)$. Then $u \in C^{2, \alpha}(\bar{\Omega})$, where $\alpha=\min \{\gamma, p, r\}$.

Proof. We only sketch the main points. First remark that since $0<r<d /(d-2)$, a Moser iteration as in [16] gives that $u \in L^{\infty}(\Omega)$ (the size of $p$ is unimportant since the corresponding term appears with a minus sign). Thus, it follows that $u$ is a weak solution to a problem of the type

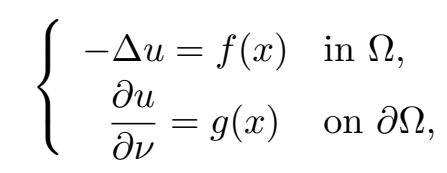

where $f \in L^{\infty}(\Omega)$ and $g \in L^{\infty}(\partial \Omega)$. The $L^{p}$ estimates of [1] (Theorem 15.2) give that $u \in W^{2, q}(\Omega)$ for every $q>1$, and the Morrey embedding provides $u \in C^{1, \beta}(\bar{\Omega})$ for some $\beta \in(0,1)$. Now it is easy to conclude $u \in C^{2, \alpha}(\bar{\Omega})$ with the help of Theorem 6.31 in $[24]$.

We now recall some well-known facts about the eigenvalue problem

$$
\left\{\begin{array}{cl}
-\Delta u+m(x) u=\lambda u & \text { in } \Omega, \\
\frac{\partial u}{\partial \nu}+h(x) u=0 & \text { on } \partial \Omega,
\end{array}\right.
$$

where $m \in L^{\infty}(\Omega), h \in C^{1}(\partial \Omega)$ (actually a little less regularity would be enough for most properties). As usual when dealing with positive solutions to nonlinear problems, we are only interested in principal eigenvalues, i.e., eigenvalues which have an associated positive eigenfunction. 
Lemma 2.2. Assume $m \in L^{\infty}(\Omega)$ and $h \in C^{1}(\partial \Omega)$. Then problem (2.1) admits a unique principal eigenvalue, which will be denoted by $\lambda_{1}(-\Delta+m, N+h)$. Moreover, this eigenvalue is simple, and any positive eigenfunction, $\varphi$, verifies $\varphi \in C^{1, \gamma}(\bar{\Omega}) \cap H^{2}(\Omega)$. In addition, $\lambda_{1}(-\Delta+m, N+h)$ is separately increasing in $m$ and $h$ and verifies

$$
\begin{aligned}
& \lim _{K \rightarrow-\infty} \lambda_{1}(-\Delta+m, N+K)=-\infty, \\
& \lim _{K \rightarrow \infty} \lambda_{1}(-\Delta+m, N+K)=\lambda_{1}(-\Delta+m, D),
\end{aligned}
$$

where $\lambda_{1}(-\Delta+m, D)$ stands for the principal eigenvalue of $-\Delta+m$ with homogeneous Dirichlet boundary conditions.

Proof. The existence of the principal eigenvalue and its simplicity are well-known, see for instance Theorem 2.2 in [2] or Lemma 7 in [22]. The fact that $\lambda_{1}(-\Delta+m, N+h)$ is separately increasing in $m$ and $h$, and (2.2) follow by [11] (see Propositions 3.3 and 3.5 and Theorem 9.1). See also Lemma 8 in [22].

We close this section with some preliminary estimates for solutions to (1.1). The first one is a pointwise lower estimate for all solutions when $p>1$ and $\lambda>0$.

Lemma 2.3. Assume that $p>1$ and $r>0$. Then, if $u$ is a positive solution to (1.1) with $\lambda>0$, we have

$$
u>\lambda^{1 /(p-1)} .
$$

Proof. It is clear that if $u$ is a positive solution to (1.1), then it is supersolution of the problem

$$
\left\{\begin{aligned}
-\Delta v & =\lambda v-v^{p} & & \text { in } \Omega, \\
\frac{\partial v}{\partial \nu} & =0 & & \text { on } \partial \Omega .
\end{aligned}\right.
$$

Moreover, $\underline{u}=\varepsilon>0$ is a subsolution of (2.4) for small $\varepsilon$. Since for $\lambda>0, \lambda^{1 /(p-1)}$ is the unique positive solution to (2.4), the result follows.

Finally, we obtain a priori bounds for the solutions to (1.1). The proof is based on a blow-up argument, as in [23]. We only sketch the proof and refer the reader to the proof of Theorem 5.1, where the details are carried out in a similar situation.

Lemma 2.4. Assume that $1 \leq p<2 r-1$ and $1<r<d /(d-2)$. For every compact interval $I \subset J$ with $J=\left(-\infty, \lambda_{1}(-\Delta+1, N)\right)$ if $p=1$ and $J=\mathbb{R}$ if $p>1$, there exists a positive constant $C$ such that every solution $(\lambda, u)$ of (1.1) with $\lambda \in I$ verifies

$$
\|u\|_{\infty} \leq C .
$$

Proof. We first claim that if $u$ is a solution to (1.1) then its maximum in $\bar{\Omega}$ is attained at $\partial \Omega$. Indeed, if $p=1$ we have that $-\Delta u=(\lambda-1) u \leq 0$ in $\Omega$. Assume now that $p>1$; if $\lambda \leq 0$ then $-\Delta u \leq 0$ in $\Omega$ and if $\lambda>0$ by (2.3) also $-\Delta u \leq 0$ in $\Omega$. So, the claim is shown.

Now assume (2.5) does not hold. Then there exists a sequence $\left\{\lambda_{j}\right\} \subset I$ with corresponding solutions $\left\{u_{j}\right\}$ such that $M_{j}=\left\|u_{j}\right\|_{\infty} \rightarrow+\infty$ as $j \rightarrow \infty$. Let $x_{j} \in \partial \Omega$ be a point where $M_{j}$ is attained. By the compactness of $\partial \Omega$, we can assume that $x_{j} \rightarrow x_{0} \in \partial \Omega$. Let

$$
v_{j}(y)=\frac{u_{j}\left(x_{j}+M_{j}^{1-r} y\right)}{M_{j}},
$$


defined in $\Omega_{j}=\left\{y \in \mathbb{R}^{d}: x_{j}+M_{j}^{1-r} y \in \Omega\right\}$. Observe that $0 \leq v_{j} \leq 1$ and $v_{j}(0)=1$, while $\Omega_{j} \rightarrow \mathbb{R}_{+}^{d}$. It is easily seen that

$$
\left\{\begin{aligned}
-\Delta v_{j} & =M_{j}^{2(1-r)} \lambda_{j} v_{j}-M_{j}^{p-2 r+1} v_{j}^{p} & & \text { in } \Omega_{j}, \\
\frac{\partial v_{j}}{\partial \nu} & =v_{j}^{r} & & \text { on } \partial \Omega_{j} .
\end{aligned}\right.
$$

By elliptic regularity $v_{j}$ is bounded in $C_{\text {loc }}^{2, \alpha}\left(\overline{\mathbb{R}}_{+}^{d}\right), \alpha \in(0,1)$. Therefore, passing to the limit through a subsequence we get a solution $0<v \leq 1$ of

$$
\begin{cases}\Delta v=0 & \text { in } \mathbb{R}_{+}^{d} \\ \frac{\partial v}{\partial \nu}=v^{r} & \text { on } \partial \mathbb{R}_{+}^{d} .\end{cases}
$$

Thanks to Theorem 1.2 of [27], this problem does not admit any positive solution. This contradiction proves the validity of (2.5).

\section{Bifurcations from zero and infinity}

We are dealing first with bifurcation from infinity for problem (1.1), see [36]. In [40] a similar result was proved when the nonlinearities are asymptotically linear, and in [7] when the nonlinearity and the bifurcation parameter appear on the boundary (see also [21]). We omit the proof here and refer to those papers for the details.

Proposition 3.1. Assume $r<1=p$ (resp. $p<1=r$ ). There exists an unbounded continuum $\mathcal{C}_{\infty} \subset \mathbb{R} \times C(\bar{\Omega})$ of nonnegative and nontrivial solutions to (1.1) bifurcating from infinity at $\lambda=\lambda_{1}(-\Delta+1, N)$ (resp. at $\lambda=\lambda_{1}(-\Delta, N-1)$ ). Moreover, this is the unique bifurcation point from infinity. Furthermore, if $\delta_{0}>0$ is small enough and $\mathcal{N}=\left[\lambda_{1}-\delta_{0}, \lambda_{1}+\delta_{0}\right] \times\left\{u \in C(\bar{\Omega}):\|u\|_{\infty} \geq 1\right\}$ with $\lambda_{1}=\lambda_{1}(-\Delta+1, N)$ (resp. $\left.\lambda_{1}=\lambda_{1}(-\Delta, N-1)\right)$, then either

1. $\mathcal{C}_{\infty} \backslash \mathcal{N}$ is bounded in $\mathbb{R} \times C(\bar{\Omega})$ in which case $\mathcal{C}_{\infty} \backslash \mathcal{N}$ meets the set $\{(\lambda, 0): \lambda \in \mathbb{R}\}$, or

2. $\mathcal{C}_{\infty} \backslash \mathcal{N}$ is unbounded in $\mathbb{R} \times C(\bar{\Omega})$.

The following result is related to bifurcation from the trivial solution, see [32]. Here, we say that in the bifurcation point $\left(\lambda_{1}, 0\right)$ the bifurcation direction is subcritical (resp. supercritical) if for every sequence $\left\{\left(\lambda_{j}, u_{j}\right)\right\}$ of positive solutions to (1.1) with $\lambda_{j} \rightarrow \lambda_{1}$ and $\left\|u_{j}\right\|_{\infty} \rightarrow 0$ as $j \rightarrow+\infty$, we have $\lambda_{j}<\lambda_{1}$ (resp. $\lambda_{j}>\lambda_{1}$ ) for large $j$.

Proposition 3.2. Assume $p \geq 1$ and $r>1$. There exists an unbounded continuum $\mathcal{C}_{0} \subset \mathbb{R} \times C(\bar{\Omega})$ of positive solutions to (1.1) emanating from the trivial solution at $\lambda=$ $\lambda_{1}(-\Delta+1, N)$ when $p=1$ or at $\lambda=0$ when $p>1$. Moreover, this is the unique bifurcation point from the trivial solution, and with respect to the bifurcation direction:

1. if $p=1<r$, then the bifurcation direction is subcritical;

2. if $1<p<r$ (resp. $p>r$ ) then the bifurcation direction is supercritical (resp. subcritical); 
3. if $p=r$ then the bifurcation direction is supercritical (resp. subcritical) for $|\Omega|>|\partial \Omega|$ (resp. $|\Omega|<|\partial \Omega|$ ).

Proof. The existence of the unbounded continuum $\mathcal{C}_{0}$ is proved in [32]. We now show the bifurcation direction in Cases 2 and 3 (the remaining case can be proved similarly). Take a sequence of solutions $\left(\lambda_{j}, u_{j}\right)$ such that $\lambda_{j} \rightarrow 0$ and $\left\|u_{j}\right\|_{\infty} \rightarrow 0$ as $j \rightarrow+\infty$. We integrate the equation (1.1), to obtain

$$
-\int_{\partial \Omega} u_{j}^{r}+\int_{\Omega} u_{j}^{p}=\lambda_{j} \int_{\Omega} u_{j}
$$

Now we divide by $\left\|u_{j}\right\|_{\infty}^{p}$ :

$$
-\left\|u_{j}\right\|_{\infty}^{r-p} \int_{\partial \Omega}\left(\frac{u_{j}}{\left\|u_{j}\right\|_{\infty}}\right)^{r}+\int_{\Omega}\left(\frac{u_{j}}{\left\|u_{j}\right\|_{\infty}}\right)^{p}=\lambda_{j}\left\|u_{j}\right\|_{\infty}^{1-p} \int_{\Omega} \frac{u_{j}}{\left\|u_{j}\right\|_{\infty}}
$$

and take into account that $u_{j} /\left\|u_{j}\right\|_{\infty} \rightarrow 1$ in $C(\bar{\Omega})$ (cf. [32]). Thus we deduce that if $1<p<r$, then $\lambda_{j}>0$ for large $j$, while if $p>r, \lambda_{j}<0$ for large $j$, which proves 2 . When $p=r$, the left-hand side of (3.1) converges to $-|\partial \Omega|+|\Omega|$. Thus $\operatorname{sgn}\left(\lambda_{j}\right)=\operatorname{sgn}(|\Omega|-|\partial \Omega|)$ for large $j$ and $|\Omega| \neq|\partial \Omega|$, which proves 3 .

\section{Proof of the main results}

We now turn to prove our theorems. For the sake of clarity, we include all the stability results in a single preliminary statement.

Lemma 4.1. Let $u_{0}$ be a positive solution to (1.1).

1. If $p \geq 1$ and $r \leq 1$ and $(p, r) \neq(1,1)$, then $u_{0}$ is stable.

2. If $p=1$ and $r>1$, then $u_{0}$ is unstable.

3. If $1<p \leq r$ and $\lambda \leq 0$, then $u_{0}$ is unstable.

Proof. We have to ascertain the sign of $\lambda_{1}\left(-\Delta-\lambda+p u_{0}^{p-1}, N-r u_{0}^{r-1}\right)$. For that, it is well known (see for instance Lemma 2.2 in [18]) that this eigenvalue is positive (resp. negative) if there exists a strict supersolution (resp. subsolution), that is, a positive function $v$ such that

$$
\left\{\begin{array}{cl}
\left(-\Delta-\lambda+p u_{0}^{p-1}\right) v \geq 0 \quad(\text { resp. } \leq 0) & \text { in } \Omega \\
\frac{\partial v}{\partial \nu}-r u_{0}^{r-1} v \geq 0 \quad(\text { resp. } \leq 0) & \text { on } \partial \Omega,
\end{array}\right.
$$

and at least one of the inequalities is strict. Observe that taking $v=u_{0}$ we have

$$
\left\{\begin{array}{cl}
-\Delta u_{0}-\lambda u_{0}+p u_{0}^{p}=(p-1) u_{0}^{p} & \text { in } \Omega, \\
\frac{\partial u_{0}}{\partial \nu}-r u_{0}^{r}=(1-r) u_{0}^{r} & \text { on } \partial \Omega,
\end{array}\right.
$$

whence we deduce the first and second paragraphs.

For the last paragraph, take $v=u_{0}^{q}$, with $1<p \leq q \leq r$. We have that

$$
\frac{\partial v}{\partial \nu}-r u_{0}^{r-1} v=(q-r) u_{0}^{q+r-1} \leq 0 \quad \text { on } \partial \Omega,
$$


and in $\Omega$,

$$
\left(-\Delta-\lambda+p u_{0}^{p-1}\right) v=q(1-q) u_{0}^{q-2}\left|\nabla u_{0}\right|^{2}+\lambda u_{0}^{q}(q-1)+u_{0}^{p+q-1}(p-q)<0 .
$$

This concludes the proof.

We are showing a uniqueness result we need in Theorems 1.1 and 1.2. It is well-known for linear boundary conditions, see [9] and [26] for example. We include the sketch of the proof for the reader's convenience, see also [22], [33] and [34].

Lemma 4.2. Consider the problem

$$
\left\{\begin{aligned}
-\Delta u & =f(x, u) & & \text { in } \Omega, \\
\frac{\partial u}{\partial \nu} & =g(x, u) & & \text { on } \partial \Omega .
\end{aligned}\right.
$$

Assume $f \in C^{1}(\bar{\Omega} \times[0,+\infty))$ and $g \in C^{1}(\partial \Omega \times[0,+\infty))$ and that

$$
t \mapsto \frac{f(x, t)}{t}, \quad t \mapsto \frac{g(x, t)}{t} \text { are nonincreasing functions in } t>0 \text {, }
$$

and at least one of them is a decreasing function. Then, problem (4.1) admits at most one positive solution.

Proof. Thanks to the regularity of the nonlinearities, we can infer that any solution $u$ verifies that $\inf _{\bar{\Omega}} u>0$. Take two positive solutions $u_{1}$ and $u_{2}$. Then

$$
\begin{aligned}
& \int_{\Omega}\left(\frac{f\left(x, u_{1}\right)}{u_{1}}-\frac{f\left(x, u_{2}\right)}{u_{2}}\right)\left(u_{1}^{2}-u_{2}^{2}\right)=\int_{\Omega}\left(-\frac{\Delta u_{1}}{u_{1}}+\frac{\Delta u_{2}}{u_{2}}\right)\left(u_{1}^{2}-u_{2}^{2}\right) \\
& =\int_{\Omega} u_{1}^{2}\left|\nabla\left(\frac{u_{2}}{u_{1}}\right)\right|^{2}+u_{2}^{2}\left|\nabla\left(\frac{u_{1}}{u_{2}}\right)\right|^{2}+\int_{\partial \Omega}\left(\frac{g\left(x, u_{2}\right)}{u_{2}}-\frac{g\left(x, u_{1}\right)}{u_{1}}\right)\left(u_{1}^{2}-u_{2}^{2}\right),
\end{aligned}
$$

and so we can deduce that $u_{1}=u_{2}$.

Remark 4.3. Observe that the regularity of the functions $f(x, \cdot)$ and $g(x, \cdot)$ are only required to prove that $c=\inf _{\bar{\Omega}} u>0$, for any positive solution $u$ of (4.1), and so $f(x, u) / u$ and $g(x, u) / u$ are well-defined.

We now come to the proof of the theorems.

\subsection{Proof of Theorem 1.1}

We divide the proof in four cases, according to whether $r=1$ and $p>1$ or $p<1$, and $p=1, r>1$ or $r<1$.

\subsubsection{Case $r=1<p$}

We begin by showing that $\lambda>\lambda_{1}(-\Delta, N-1)$ is necessary for the existence of positive solutions. Denote by $\varphi_{1}$ the positive eigenfunction associated to $\lambda_{1}(-\Delta, N-1)$. Then, multiplying (1.1) by $\varphi_{1}$ and integrating by parts, we get

$$
\left(\lambda_{1}(-\Delta, N-1)-\lambda\right) \int_{\Omega} u \varphi_{1}=-\int_{\Omega} u^{p} \varphi_{1}
$$


Thus $\lambda>\lambda_{1}(-\Delta, N-1)$. To show the existence of solutions when $\lambda>\lambda_{1}(-\Delta, N-1)$, we use the method of sub and supersolutions. The function $\bar{u}:=M \varphi_{1}$ is a supersolution of (1.1) provided we take

$$
M=\frac{\left(\lambda-\lambda_{1}(-\Delta, N-1)\right)^{1 /(p-1)}}{\delta_{0}},
$$

with $0<\delta_{0}=\min _{x \in \bar{\Omega}} \varphi_{1}(x)$. On the other hand, $\underline{u}:=\varepsilon \varphi_{1}$ for $\varepsilon>0$ is subsolution of (1.1) provided that

$$
\varepsilon^{p-1} \varphi_{1}^{p-1} \leq \lambda-\lambda_{1}(-\Delta, N-1) \quad \text { in } \Omega .
$$

It suffices to take $\varepsilon>0$ small enough such that $\underline{u} \leq \bar{u}$ and the existence of a positive solution follows. The uniqueness follows directly by Lemma 4.2.

Now, thanks to uniqueness and the way the supersolution was built, see (4.2), we conclude that

$$
\lim _{\lambda \backslash \lambda_{1}(-\Delta, N-1)}\left\|u_{\lambda}\right\|_{\infty}=0
$$

and by Lemma 2.3,

$$
\lim _{\lambda \nearrow+\infty}\left\|u_{\lambda}\right\|_{\infty}=+\infty .
$$

The stability follows by Lemma 4.1, paragraph 1.

\subsubsection{Case $p<1=r$}

The necessity of $\lambda>\lambda_{1}(-\Delta, N-1)$ to have nonnegative and nontrivial solutions follows in a similar way as before. Now, we can apply Proposition 3.1 and so an unbounded continuum $\mathcal{C}_{\infty}$ of positive solutions to (1.1) bifurcates at $\lambda=\lambda_{1}(-\Delta, N-1)$. It suffices to show that this continuum does not meet the set $\{(\lambda, 0): \lambda \in \mathbb{R}\}$. Assume that there exists a sequence $\left(\lambda_{j}, u_{j}\right)$ of solutions to (1.1) such that $\lambda_{j} \rightarrow \lambda_{0} \geq \lambda_{1}(-\Delta, N-1)$ and $\left\|u_{j}\right\|_{\infty} \rightarrow 0$ as $j \rightarrow \infty$. Take $M \geq \lambda_{j}-\lambda_{1}(-\Delta, N-1)$. For $j$ large enough, we have that $u_{j}^{p}>M u_{j}$ and so

$$
\left\{\begin{array}{rlrl}
-\Delta u_{j}<\left(\lambda_{j}-M\right) u_{j} & & \text { in } \Omega, \\
\frac{\partial u_{j}}{\partial \nu} & =u_{j} & & \text { on } \partial \Omega,
\end{array}\right.
$$

which implies $\lambda_{1}\left(-\Delta-\lambda_{j}+M, N-1\right)<0$, that is, $\lambda_{1}(-\Delta, N-1)-\lambda_{j}+M<0$, a contradiction. Finally, by Theorem 5.1 in Section 5 we have that

$$
\lim _{\lambda \nearrow+\infty}\left\|u_{\lambda}\right\|_{\infty}=0
$$

for every family $\left\{u_{\lambda}\right\}$ of nonnegative solutions.

\subsubsection{Case $p=1<r<d /(d-2)$}

Consider $\varphi_{1}$ a positive eigenfunction associated to $\lambda_{1}(-\Delta+1, N)$. Multiplying (1.1) by $\varphi_{1}$ and integrating we get

$$
-\int_{\partial \Omega} u^{r} \varphi_{1}=\left(\lambda-\lambda_{1}(-\Delta+1, N) \int_{\Omega} u \varphi_{1},\right.
$$


and hence $\lambda<\lambda_{1}(-\Delta+1, N)$. Now, we can apply Proposition 3.2 and deduce the existence of an unbounded continuum $\mathcal{C}_{0}$ bifurcating subcritically at $\lambda=\lambda_{1}(-\Delta+1, N)$. Thanks to the a priori bounds (Lemma 2.4) we deduce the existence of at least a positive solution for every $\lambda<\lambda_{1}(-\Delta+1, N)$. By Lemma 4.1, paragraph 2, every solution is unstable. Finally, by Theorem 5.3 in Section 5 we have that

$$
\lim _{\lambda \searrow-\infty}\left\|u_{\lambda}\right\|_{\infty}=+\infty
$$

for any family $\left\{u_{\lambda}\right\}$ of positive solutions.

\subsubsection{Case $r<1=p$}

Again, $\lambda<\lambda_{1}(-\Delta+1, N)$ is necessary to have positive solutions. By Proposition 3.1, there exists a unbounded continuum $\mathcal{C}_{\infty}$ of positive solutions bifurcating from infinity at $\lambda=\lambda_{1}(-\Delta+1, N)$.

Assume that there exists a sequence $\left(\lambda_{j}, u_{j}\right)$ of solutions to (1.1) such that $\lambda_{j} \rightarrow$ $\lambda_{0} \leq \lambda_{1}(-\Delta+1, N)$ and $\left\|u_{j}\right\|_{\infty} \rightarrow 0$ as $j \rightarrow \infty$. Take $M>0$ large enough so that $-\lambda_{j}+\lambda_{1}(-\Delta+1, N-M) \leq 0$, which is possible thanks to (2.2) in Lemma 2.2. For this value of $M$ and large $j$ we have that $u_{j}^{r}>M u_{j}$, and so

$$
\left\{\begin{array}{cl}
\left(-\Delta-\lambda_{j}+1\right) u_{j}=0 & \text { in } \Omega, \\
\frac{\partial u_{j}}{\partial \nu}-M u_{j}>0 & \text { on } \partial \Omega .
\end{array}\right.
$$

Hence $\lambda_{1}\left(-\Delta+1-\lambda_{j}, N-M\right)>0$, a contradiction. This completes the proof of the existence of solutions. The uniqueness and stability follow by Lemmas 4.2 and 4.1, respectively. Observe that although $u^{r}$ is not a $C^{1}$ function in $[0, \infty)$, any solution is in fact strictly positive, and thus both results can be applied, see Remark 4.3. Moreover, by Theorem 5.3 we have that

$$
\lim _{\lambda \searrow-\infty}\left\|u_{\lambda}\right\|_{\infty}=0
$$

for the unique positive solution.

\subsection{Proof of Theorem 1.2}

Now $0<r<1<p$. We are using the method of sub and supersolutions to prove existence. For fixed $\lambda \in \mathbb{R}$, we choose $K_{0} \in \mathbb{R}$ so that $\lambda_{1}\left(-\Delta, N-K_{0}\right)<\lambda$ (this is possible according to (2.2) in Lemma 2.2). Define $\underline{u}:=\varepsilon \varphi_{1}$ with $\varepsilon>0$ and $\varphi_{1}$ the positive eigenfunction associated to $\lambda_{1}\left(-\Delta, N-K_{0}\right)$. Then, $\underline{u}$ is subsolution of (1.1) provided that

$$
\begin{array}{cl}
\varepsilon^{p-1} \varphi_{1}^{p-1} \leq \lambda-\lambda_{1}\left(-\Delta, N-K_{0}\right) & \text { in } \Omega, \\
K_{0}\left(\varepsilon \varphi_{1}\right)^{1-r} \leq 1 & \text { on } \partial \Omega .
\end{array}
$$

Thus it suffices to take $\varepsilon$ small enough. To build the supersolution, take $\bar{u}:=M \varphi_{1}>0$ with a large $M$. Then $\bar{u}$ will be a supersolution of (1.1) if

$$
\begin{array}{cl}
M^{p-1} \varphi_{1}^{p-1} \geq \lambda-\lambda_{1}\left(-\Delta, N-K_{0}\right) & \text { in } \Omega, \\
K_{0}\left(M \varphi_{1}\right)^{1-r} \geq 1 & \text { on } \partial \Omega,
\end{array}
$$


which is true if $M$ is large enough.

The uniqueness and stability follow again by Lemmas 4.2 and 4.1. Moreover, thanks to Theorem 5.3 and Lemma 2.3, we have

$$
\lim _{\lambda \searrow-\infty}\left\|u_{\lambda}\right\|_{\infty}=0, \quad \quad \lim _{\lambda \nearrow+\infty}\left\|u_{\lambda}\right\|_{\infty}=+\infty .
$$

\subsection{Proof of Theorem 1.3}

To prove the existence of solutions in this case we use variational arguments. Thus we consider in $H^{1}(\Omega)$ the functional whose critical points coincide with weak solutions to (1.1):

$$
F(u)=\frac{1}{2} \int_{\Omega}|\nabla u|^{2}-\frac{\lambda}{2} \int_{\Omega}\left|u_{+}\right|^{2}+\frac{1}{p+1} \int_{\Omega}|u|^{p+1}-\frac{1}{r+1} \int_{\partial \Omega}\left|u_{+}\right|^{r+1},
$$

where $u_{+}=\max \{u, 0\}$.

Since $r$ is subcritical, $r<d /(d-2)$, it is well-known that $F$ is well defined and $C^{1}$ in $H^{1}(\Omega)$. By means of the Mountain Pass Theorem (see [5]), we are showing that there exists at least a nontrivial critical point $u \in H^{1}(\Omega)$ of $F$, which will be a nontrivial weak solution to (1.1). According to Lemma 2.1, $u \in C^{2, \alpha}(\bar{\Omega})$ will be a classical solution to (1.1). Thus we only have to prove that the geometric conditions to apply the Mountain Pass Theorem hold:

Lemma 4.4. Assume that $0<p<1<r<d /(d-2)$. Then:

1. There exists a constant $c$ such that for $r$ small enough, $F(u) \geq c r^{3}$ if $\|u\|_{H^{1}(\Omega)}=r$.

2. There exists $v_{0}$ with large $H^{1}(\Omega)$-norm such that $F\left(v_{0}\right)<0$.

3. F verifies the Palais-Smale condition.

Proof. 1. We argue by contradiction. Assume that there exists a sequence $u_{n}$ such that

$$
\left\|u_{n}\right\|_{H^{1}(\Omega)}=r_{n} \rightarrow 0 \quad \text { and } \quad \limsup _{n \rightarrow \infty} \frac{F\left(u_{n}\right)}{r_{n}^{3}} \leq 0 .
$$

Let $v_{n}=u_{n} / r_{n}$. Since $\left\|v_{n}\right\|_{H^{1}(\Omega)}=1$ we can extract a subsequence such that

$$
\begin{aligned}
& v_{n} \rightarrow v_{0} \\
& v_{n} \rightarrow v_{0} \quad \text { weakly in } H^{1}(\Omega), \\
& \text { strongly in } L^{2}(\Omega), L^{p+1}(\Omega), L^{r+1}(\partial \Omega) .
\end{aligned}
$$

From (4.3) we obtain

$$
\limsup _{n \rightarrow \infty} \frac{1}{r_{n}}\left(\frac{1}{2} \int_{\Omega}\left|\nabla v_{n}\right|^{2}-\frac{\lambda}{2} \int_{\Omega}\left|\left(v_{n}\right)_{+}\right|^{2}+\frac{r_{n}^{p-1}}{p+1} \int_{\Omega}\left|v_{n}\right|^{p+1}-\frac{r_{n}^{r-1}}{r+1} \int_{\partial \Omega}\left|\left(v_{n}\right)_{+}\right|^{r+1}\right) \leq 0 .
$$

Since $p<1$, the weak limit $v_{0}$ verifies

$$
\int_{\Omega}\left|v_{0}\right|^{p+1}=0
$$


and so $v_{0} \equiv 0$ in $\Omega$. Going back to (4.4), we get

$$
\int_{\Omega}\left|\nabla v_{n}\right|^{2} \rightarrow 0
$$

a contradiction with $\left\|v_{n}\right\|_{H^{1}(\Omega)}=1$.

2. We take any function $v$ such that $v \not \equiv 0$ on $\partial \Omega$ and observe that

$$
\lim _{t \rightarrow+\infty} F(t v)=-\infty
$$

Hence it suffices with setting $v_{0}=t v$ for a large $t$.

3. Let $u_{n}$ be a Palais-Smale sequence, that is a sequence such that

$$
\left|F\left(u_{n}\right)\right| \leq C \quad \text { and } \quad F^{\prime}\left(u_{n}\right) \rightarrow 0
$$

We have to prove that it contains a strongly convergent subsequence. To this end let us first check that it is bounded. Assume that this is not the case, that is, passing to a subsequence $\left\|u_{n}\right\|_{H^{1}(\Omega)} \rightarrow \infty$. Let

$$
v_{n}=\frac{u_{n}}{\left\|u_{n}\right\|_{H^{1}(\Omega)}} .
$$

Since $v_{n}$ is bounded in $H^{1}(\Omega)$ there exists a subsequence (that we still denote by $v_{n}$ ) such that

$$
\begin{aligned}
& v_{n} \rightarrow v_{0} \\
& v_{n} \rightarrow v_{0} \quad \text { weakly in } H^{1}(\Omega), \\
& \text { strongly in } L^{2}(\Omega), L^{p+1}(\Omega), L^{r+1}(\partial \Omega) .
\end{aligned}
$$

On the other hand, since $F\left(u_{n}\right)$ is bounded and $F^{\prime}\left(u_{n}\right) \rightarrow 0$ we get

$$
\begin{aligned}
\frac{F\left(u_{n}\right)}{\left\|u_{n}\right\|_{H^{1}(\Omega)}}-\frac{1}{2}\left\langle F^{\prime}\left(u_{n}\right), v_{n}\right\rangle= & \left(\frac{1}{p+1}-\frac{1}{2}\right)\left\|u_{n}\right\|_{H^{1}(\Omega)}^{p} \int_{\Omega}\left|v_{n}\right|^{p+1} \\
& +\left(\frac{1}{2}-\frac{1}{r+1}\right)\left\|u_{n}\right\|_{H^{1}(\Omega)}^{r} \int_{\partial \Omega}\left|\left(v_{n}\right)_{+}\right|^{r+1} \rightarrow 0 .
\end{aligned}
$$

Hence

$$
v_{0} \equiv 0 \quad \text { in } \Omega .
$$

In addition,

$$
\left\langle F^{\prime}\left(u_{n}\right), \frac{u_{n}}{\left\|u_{n}\right\|_{H^{1}(\Omega)}^{2}}\right\rangle \rightarrow 0
$$

That is,

$$
\int_{\Omega}\left|\nabla v_{n}\right|^{2}-\lambda \int_{\Omega}\left|\left(v_{n}\right)_{+}\right|^{2}+\left\|u_{n}\right\|_{H^{1}(\Omega)}^{p-1} \int_{\Omega}\left|v_{n}\right|^{p+1}-\left\|u_{n}\right\|_{H^{1}(\Omega)}^{r-1} \int_{\partial \Omega}\left|\left(v_{n}\right)_{+}\right|^{q+1} \rightarrow 0,
$$

and taking into account (4.5), we get that

$$
\int_{\Omega}\left|\nabla v_{n}\right|^{2} \rightarrow 0
$$


again a contradiction. Thus $\left\{u_{n}\right\}$ is bounded in $H^{1}(\Omega)$. We may pass to a subsequence which verifies

$$
\begin{aligned}
& u_{n} \rightarrow u_{0} \\
& u_{n} \rightarrow u_{0} \quad \text { weakly in } H^{1}(\Omega), \\
& \text { strongly in } L^{2}(\Omega), L^{p+1}(\Omega), L^{r+1}(\partial \Omega) .
\end{aligned}
$$

Since $\left\langle F^{\prime}\left(u_{n}\right), u_{n}\right\rangle \rightarrow 0,\left\langle F^{\prime}\left(u_{n}\right), u_{0}\right\rangle \rightarrow 0$, we have

$$
\int_{\Omega}\left|\nabla u_{n}\right|^{2}-\lambda \int_{\Omega}\left|\left(u_{n}\right)_{+}\right|^{2}+\int_{\Omega}\left|u_{n}\right|^{p+1}-\int_{\partial \Omega}\left|\left(u_{n}\right)_{+}\right|^{r+1} \rightarrow 0
$$

and

$$
\int_{\Omega} \nabla u_{n} \cdot \nabla u_{0}-\lambda \int_{\Omega}\left(u_{n}\right)_{+} u_{0}+\int_{\Omega}\left|u_{n}\right|^{p-1} u_{n} u_{0}-\int_{\partial \Omega}\left|\left(u_{n}\right)_{+}\right|^{r-1} u_{n} u_{0} \rightarrow 0 .
$$

And thanks to the weak convergence of $u_{n}$ we arrive at

$$
\lim _{n \rightarrow \infty} \int_{\Omega}\left|\nabla u_{n}\right|^{2}=\int_{\Omega}\left|\nabla u_{0}\right|^{2}
$$

which proves that $u_{n}$ converges strongly to $u_{0}$. This completes the proof.

\subsection{Proof of Theorem 1.4}

The proof of this theorem is much more involved than that of the previous ones. First, we state the sweeping method of Serrin ([39], page 12) which allows to obtain some a priori bounds for the solutions of (1.1).

Lemma 4.5. Let $u$ be a solution of (1.1). Assume that there exists a family $\bar{u}_{M} \in C^{1}(\bar{\Omega})$ of strict supersolutions of (1.1) for $M \in\left[M_{0}, M_{1}\right]$ such that $\bar{u}_{M}$ is a continuos and increasing function in $M$ and $u \leq \bar{u}_{M_{1}}$ in $\bar{\Omega}$. Then, it holds

$$
u \leq \bar{u}_{M_{0}} \quad \text { in } \bar{\Omega} \text {. }
$$

Now, we state and prove a non-existence result.

Lemma 4.6. Assume $p, r>1$. Then:

1. If $p=r$ and $|\Omega| \leq|\partial \Omega|$, problem (1.1) does not have positive solutions for $\lambda \geq 0$.

2. Assume that $p \leq 2 r-1$. Then, there exists $\Lambda_{1}>0$ such that problem (1.1) does not have positive solutions for $\lambda \geq \Lambda_{1}$.

3. Assume that $p>2 r-1$. Then, there exists $\Lambda_{2}<0$ such that problem (1.1) does not have positive solutions for $\lambda \leq \Lambda_{2}$.

Proof. 1. Let $u$ be a positive solution to (1.1) with $p=r$. Then, multiplying (1.1) by $1 / u^{r}$ and integrating by parts, we get

$$
-r \int_{\Omega} u^{-r-1}|\nabla u|^{2}-|\partial \Omega|+|\Omega|=\lambda \int_{\Omega} u^{1-r}
$$


The first paragraph follows.

2. Assume that there exists a sequence $\lambda_{n} \nearrow \infty$ with corresponding solutions $u_{n}$ of (1.1). Consider the parabolic problem

$$
\begin{cases}w_{t}-\Delta w=-w^{p} & \text { in } \Omega \times(0, T), \\ \frac{\partial w}{\partial \nu}=w^{r} & \text { on } \partial \Omega \times(0, T), \\ w(x, 0)=w_{0} & \text { in } \Omega .\end{cases}
$$

We know by Theorem 2.3 in [6], that if $p \leq 2 r-1$ then all positive solutions to (4.6) blow-up in a finite time $T>0$ provided $\inf _{\bar{\Omega}} w_{0}$ is large enough. If we prove that $u_{n}$ is a supersolution of (4.6) for large $n$, then $u_{n}(x)>w(x, t)$ for all $t \in(0, T)$ which is clearly a contradiction. Observe that $u_{n}$ is supersolution of (4.6) if $u_{n}>w_{0}$. By Lemma 2.3, we have $u_{n}>\lambda_{n}^{1 /(p-1)}$. Thus for large enough $n$, we may set $w_{0}=\lambda_{n}^{\frac{1}{p-1}}$, which concludes the proof of the second paragraph.

3. Assume now that $p>2 r-1$. We want to show that for $\lambda$ negative enough, there are no positive solutions to (1.1). First, we claim that there exists a positive function $U \in C(\bar{\Omega})$ such that

$$
u_{\lambda} \leq U
$$

for all family of positive solutions of (1.1) with $\lambda \leq 0$. Assume (4.7). Consider the problem

$$
\begin{cases}v_{t}-\Delta v=\lambda v-v^{p} & \text { in } \Omega \times(0, T), \\ \frac{\partial v}{\partial \nu}=v^{r} & \text { on } \partial \Omega \times(0, T), \\ v(x, 0)=v_{0}(x)>0 & \text { in } \Omega\end{cases}
$$

and denote $v\left(t ; v_{0}\right)$ its positive solution. It is clear that

$$
u_{\lambda}=v\left(t ; u_{\lambda}\right) \leq v(t ; U)
$$

and so it suffices to prove $\|v(t, U)\|_{\infty} \rightarrow 0$ as $t \rightarrow \infty$ for negative enough $\lambda$. To this aim, it suffices to construct a global supersolution of (4.8) which goes to zero at infinity. Since $p>2 r-1$, for every initial datum $w_{0} \in L^{\infty}(\Omega)$, (4.6) has a positive solution $w$, which is globally bounded (cf. [6]). Consider $\bar{v}:=e^{-\mu t} w$ for some fixed $\mu>0$. It is not hard to show that $\bar{v}$ is a supersolution of (4.8) provided that

$$
\begin{array}{cl}
w^{p-1}\left(e^{-(p-1) \mu t}-1\right)-\mu \geq \lambda & \text { in } \Omega \times(0, T), \\
e^{\mu(r-1) t} \geq 1 & \text { on } \partial \Omega \times(0, T) .
\end{array}
$$

Since $w$ is bounded, there exists $\lambda_{0}<0$ such that for $\lambda \leq \lambda_{0}$ the two inequalities hold. Thus, it remains to prove (4.7). For that, we are going to use Lemma 4.5 and so we need to construct now a family of strict supersolutions of (1.1). For the particular case $\lambda=0$ a different supersolution was used in [29] and [43]. Take

$$
\bar{u}_{M}:=M\left(\phi+M^{-\sigma}\right)^{-\beta}
$$


where $\beta=2 /(p-1), \phi$ is such that

$$
\left\{\begin{array}{cl}
-\Delta \phi=\lambda_{1} \phi & \text { in } \Omega, \\
\phi=0 & \text { on } \partial \Omega,
\end{array}\right.
$$

and $M, \sigma>0$ are to be chosen. It is clear that $\bar{u}_{M}$ is a continuous and increasing function in $M$. After some calculations, we have that $\bar{u}_{M}$ is strict supersolution of (1.1) provided that

$$
M^{p-1}-\beta(1+\beta)|\nabla \phi|^{2}-\beta\left(\phi+M^{-\sigma}\right) \lambda_{1} \phi-\lambda\left(\phi+M^{-\sigma}\right)^{2} \geq 0 \quad \text { in } \Omega
$$

and

$$
-\beta \frac{\partial \phi}{\partial \nu}>M^{r-1-\sigma[\beta(1-r)+1]} \quad \text { on } \partial \Omega \text {. }
$$

Take into account that $-\partial \phi / \partial \nu \geq c_{1}>0$ on $\partial \Omega$, it is not hard to show that there exists $M_{0}>0$ (independent of $\lambda$ ) such that for $M \geq M_{0}$ and $\lambda \leq 0$ both inequalities are satisfied, provided that (recall $p>2 r-1$ )

$$
r-1-\sigma[\beta(1-r)+1]<0
$$

that is

$$
\sigma>\frac{(p-1)(r-1)}{p-2 r+1}
$$

On the other hand, given a positive solution $u_{\lambda}$ of (1.1) with $\lambda \leq 0$, there exists a sufficiently large $M(\lambda)>M_{0}$ such that $u_{\lambda}<\bar{u}_{M(\lambda)}$. So, we can apply Lemma 4.5 and conclude that $u_{\lambda} \leq \bar{u}_{M_{0}}:=U$. This proves (4.7) and completes the proof.

We are now ready to come to the proof of Theorem 1.4.

\subsubsection{Case $p>2 r-1$}

From Proposition 3.2 it follows that there exists an unbounded continuum $\mathcal{C}_{0}$ of positive solutions bifurcating at $\lambda=0$ subcritically (observe that $p>r$ in this case).

Take $I=\left[\Lambda_{2}, K\right]$, with $K>\Lambda_{2}$ arbitrary where $\Lambda_{2}$ is given by Lemma 4.6. We have a continuous map $\bar{u}: I \rightarrow C^{1}(\bar{\Omega}), \lambda \mapsto \bar{u}(\lambda)$ where $\bar{u}(\lambda)$ is the strict supersolution of (1.1) which has been constructed above. Moreover, we have a connected set $\mathcal{C}_{0}$ such that for $\lambda_{0}$ small enough $u_{\lambda_{0}}<\bar{u}\left(\lambda_{0}\right)$ for $\left(\lambda_{0}, u_{\lambda_{0}}\right) \in \mathcal{C}_{0}$. Then by a similar reasoning to the used in [19] we obtain that $u_{\lambda}<\bar{u}(\lambda)$ for all $\left(\lambda, u_{\lambda}\right) \in \mathcal{C}_{0}$ and $\lambda \in I$. This implies that the projection on the real axis of the continuum $\mathcal{C}_{0}$ is $\left[\lambda_{2},+\infty\right)$ for some $\lambda_{2}<0$.

To complete the proof, set $\lambda_{0}:=\inf \{\lambda \in \mathbb{R}:(1.1)$ has a positive solution $\}$. Thanks to Lemma 4.6 we know that $-\infty<\lambda_{0}<0$. Now, we want to show that there exists a solution for all $\lambda \geq \lambda_{0}$. Indeed, for $\lambda>\lambda_{0}$, we can take $\lambda_{1} \in\left(\lambda_{0}, \lambda\right)$ such that the corresponding solution $u_{\lambda_{1}}$ (which exists thanks to the definition of $\lambda_{0}$ ) is subsolution of (1.1) for this $\lambda$. Again, as supersolution we can take $\bar{u}(\lambda)$. Thus there exists a solution for every $\lambda>\lambda_{0}$.

Finally, we show that there exists a solution for $\lambda=\lambda_{0}$. Take $\left(\lambda_{j}, u_{j}\right)$ a sequence of solutions such that $0>\lambda_{j}>\lambda_{0}$ and $\lambda_{j} \rightarrow \lambda_{0}$. Since we have an a priori bound for all solutions, namely $u_{j}<U$ (see (4.7)), it is standard to pass to the limit to obtain that $u_{j} \rightarrow u_{0}$ with $u_{0}$ a solution to (1.1) for $\lambda=\lambda_{0}$. Since $\lambda_{0}<0$, it cannot be a bifurcation point from the trivial solution, and hence $u_{0} \not \equiv 0$. This completes the proof. 


\subsubsection{Case $p<2 r-1$}

Thanks to Proposition 3.2, there exists an unbounded continuum $\mathcal{C}_{0}$ of positive solutions to (1.1) which emanates from zero at $\lambda=0$, and by Lemma 2.4 the solutions are bounded for bounded $\lambda$. Thus, since there are no positive solutions for large $\lambda$, we conclude the existence of $\lambda_{0} \geq 0$ such that there exists at least a positive solution to (1.1) for $\lambda<\lambda_{0}$. Moreover, (1.9) follows by Theorem 5.3 in Section 5.

Now define $\Lambda_{0}:=\sup \{\lambda \in \mathbb{R}:(1.1)$ has a positive solution $\}$. We already know that $0 \leq \Lambda_{0}<+\infty$, and clearly there are no solutions for $\lambda>\Lambda_{0}$. It remains to show that when $\Lambda_{0}>0$ there exist at least two positive solutions for all $\lambda \in\left(0, \Lambda_{0}\right)$ and one positive solution for $\lambda=\Lambda_{0}$.

We first show that a minimal positive solution exists if $\lambda \in\left(0, \Lambda_{0}\right)$. Fix such a $\lambda$. We have that there exists $\bar{\lambda} \in\left(\lambda, \Lambda_{0}\right)$ such a positive solution $u_{\bar{\lambda}}$ of (1.1) exists. It is clear that $u_{\bar{\lambda}}$ is a supersolution to (1.1) for all $\lambda \leq \bar{\lambda}$. On the other hand, $\underline{u}=\varepsilon$ is a subsolution for small $\varepsilon>0$. Thus there exists at least a positive solution for every $\lambda \in\left(0, \Lambda_{0}\right)$.

Moreover, we have that any positive solution $u_{\lambda}$ verifies $u_{\lambda}>\lambda^{1 /(p-1)}$, thanks to Lemma 2.3. Hence, the existence of a minimal solution to (1.1) follows. It will be denoted by $u_{\lambda}$.

We now show the existence of a second solution when $\lambda \in\left(0, \Lambda_{0}\right)$. We are proving for this aim that our problem is in the general setting of [3] (we refer there for the definitions to be used in the sequel). Let $P$ be the cone of positive functions of $C(\bar{\Omega})$. With the ordering induced by $P, C(\bar{\Omega})$ is an ordered Banach space with a normal cone which has nonempty interior, see Example 1.10 in [3]. Consider the interval $I=\left[-1, \Lambda_{0}+1\right]$ and let

$$
\beta>\sup _{\lambda \in I}\|u(\lambda)\|_{\infty}
$$

being $u(\lambda)$ any solution to (1.1). This is possible since we have a priori bounds for the solutions when $\lambda$ runs in finite intervals (cf. Lemma 2.4). Take $K>0$ a constant to be chosen later, so that (1.1) can be rewritten as

$$
\left\{\begin{array}{cl}
(-\Delta+K) u=(\lambda+K) u-u^{p} & \text { in } \Omega, \\
\frac{\partial u}{\partial \nu}=u^{r} & \text { on } \partial \Omega .
\end{array}\right.
$$

We want to show that solving our problem is equivalent to find fixed points of a nonlinear operator. For that, let $\mathcal{K}_{1}: C^{\alpha}(\bar{\Omega}) \mapsto C^{2, \alpha}(\bar{\Omega}), \alpha \in(0,1)$, be the operator such that $f \mapsto u=\mathcal{K}_{1} f$ where $u$ is the unique solution to

$$
\left\{\begin{array}{cl}
(-\Delta+K) u=f & \text { in } \Omega \\
\frac{\partial u}{\partial \nu}=0 & \text { on } \partial \Omega .
\end{array}\right.
$$

This operator can be extended to a linear, compact and strongly positive map, denoted again by $\mathcal{K}_{1}, \mathcal{K}_{1}: C(\bar{\Omega}) \mapsto C^{1}(\bar{\Omega})$, see Theorem 4.2 in [3]. Consider now the operator $\mathcal{K}_{2}: C^{1, \alpha}(\partial \Omega) \mapsto C^{2, \alpha}(\bar{\Omega}), g \mapsto u=\mathcal{K}_{2} g$, where $u$ is the unique solution to

$$
\left\{\begin{array}{cl}
(-\Delta+K) u=0 & \text { in } \Omega \\
\frac{\partial u}{\partial \nu}=g & \text { on } \partial \Omega .
\end{array}\right.
$$


Now, by [2], $\mathcal{K}_{2}$ can be extended to a linear compact map from $C(\partial \Omega)$ to $C(\bar{\Omega})$. It is not hard to prove that $u$ is solution to (1.1) if and only if

$$
u=F(u, \lambda)=\mathcal{K}_{1}\left((\lambda+K) u-u^{p}\right)+\mathcal{K}_{2}\left(\gamma\left(u^{r}\right)\right),
$$

where $\gamma: C(\bar{\Omega}) \mapsto C(\partial \Omega)$ is the trace operator.

Moreover, $F: C(\bar{\Omega}) \times \mathbb{R} \rightarrow C(\bar{\Omega})$ is a differentiable operator, which is compact on bounded sets, and it is strongly increasing for fixed $\lambda$ if $K$ is large enough and $u$ is restricted to bounded sets. In addition, the partial derivatives,

$$
\partial_{u} F\left(u_{0}, \lambda_{0}\right) \xi=\mathcal{K}_{1}\left((\lambda+K)-p u_{0}^{p-1}\right) \xi+\mathcal{K}_{2}\left(r \gamma\left(u_{0}^{r-1}\right)\right) \gamma(\xi)
$$

and

$$
\partial_{\lambda} F\left(u_{0}, \lambda_{0}\right) \mu=\mu \mathcal{K}_{1} u_{0}
$$

are strongly positive if $K$ is selected large enough. Indeed, observe that since $\lambda \in I$, then $\sup _{\lambda \in I}\|u(\lambda)\|_{\infty}<\beta$ for any positive solution $u(\lambda)$ of (1.1), and so $K$ can be taken large to make the partial derivatives strongly positive. Hence, $F$ satisfies hypothesis $(H)$ of [3] page 680, and so we can apply Theorem 20.9 of [3] (see the arguments after Proposition 20.8 and Theorem 7.4 in [4]) and conclude the existence of at least two positive solutions for $\lambda \in\left(0, \Lambda_{0}\right)$ and at least a positive solution for $\lambda=\Lambda_{0}$.

We quote for its use in the next section that, denoting by $\rho=r\left(u_{0}, \lambda_{0}\right)$ the spectral radius of $\partial_{u} F\left(u_{0}, \lambda_{0}\right)$, then $\rho$ satisfies

$$
\lambda_{1}\left(-\Delta+\frac{1}{\rho}\left(p u_{0}^{p-1}-\lambda_{0}\right), N+\frac{r}{\rho} u_{0}^{r-1}\right)=K\left(\frac{1}{\rho}-1\right) .
$$

\subsubsection{Case $p<r$ or $p=r$ and $|\Omega|>|\partial \Omega|$}

First of all, notice that $p<2 r-1$ in this case. Thus there exists a solution for every $\lambda<\Lambda_{0}$, for a certain $\Lambda_{0} \geq 0$. Since a supercritical bifurcation takes place at $\lambda=0$ (Proposition 3.2) we have $\Lambda_{0}>0$. Thus only the uniqueness of the stable solution for $\lambda \in\left(0, \Lambda_{0}\right)$ remains to be proved. We adapt the argument used in [25].

The following result provides us with a complete picture of the structure of the set of positive solutions near a stable or neutrally stable solution.

Lemma 4.7. Let $\left(\lambda_{0}, u_{0}\right)$ be a positive solution to (1.1) with $\lambda=\lambda_{0}$.

1. If

$$
\lambda_{1}\left(-\Delta-\lambda_{0}+p u_{0}^{p-1}, N-r u_{0}^{r-1}\right)>0,
$$

then, there exists $\varepsilon>0$ and a differentiable mapping $u: I=\left(\lambda_{0}-\varepsilon, \lambda_{0}+\varepsilon\right) \mapsto P$ such that $u\left(\lambda_{0}\right)=u_{0}$ and $(\lambda, u(\lambda))$ is a positive solution to (1.1) for each $\lambda \in I$. Moreover, the mapping $\lambda \mapsto u(\lambda)$ is increasing and there exists a neighborhood $\mathcal{V}$ of $\left(\lambda_{0}, u_{0}\right)$ in $\mathbb{R} \times P$ such that if $(\lambda, u) \in \mathcal{V}$ is a solution to $(1.1)$, then $(\lambda, u)=(\lambda, u(\lambda))$ for some $\lambda \in I$.

2. If

$$
\lambda_{1}\left(-\Delta-\lambda_{0}+p u_{0}^{p-1}, N-r u_{0}^{r-1}\right)=0,
$$

let $\Phi_{0}$ be the principal eigenfunction associated with $\lambda_{1}\left(-\Delta-\lambda_{0}+p u_{0}^{p-1}, N-r u_{0}^{r-1}\right)$. Then, there exists $\varepsilon>0$ and a twice continuously differentiable mapping $(\lambda, u): J=$ 
$(-\varepsilon, \varepsilon) \mapsto \mathbb{R} \times P$ such that $(\lambda(0), u(0))=\left(\lambda_{0}, u_{0}\right)$ and for each $s \in J,(\lambda(s), u(s))$ is a positive solution to (1.1). Moreover, $\lambda^{\prime}(0)=0, u(s)=u_{0}+s\left(\Phi_{0}+v(s)\right)$ where $v \in C^{1}((-\varepsilon, \varepsilon), C(\bar{\Omega}))$ satisfies $v(0)=0$, and finally

$$
\lambda^{\prime \prime}(0)=\frac{\int_{\Omega} p(p-1) u_{0}^{p-2} \Phi_{0}^{3}-\int_{\partial \Omega} r(r-1) u_{0}^{r-2} \Phi_{0}^{3}}{\int_{\Omega} u_{0} \Phi_{0}},
$$

for $s \simeq 0$. In addition, there exists a neighborhood $\mathcal{W}$ of $\left(\lambda_{0}, u_{0}\right)$ in $\mathbb{R} \times P$ such that if $(\lambda, u) \in \mathcal{W}$ is a solution to (1.1), then $(\lambda, u)=(\lambda(s), u(s))$ for some $s \in J$. Also,

$$
\operatorname{sgn} \lambda^{\prime}(s)=\operatorname{sgn} \lambda_{1}\left(-\Delta-\lambda(s)+p u(s)^{p-1}, N-r u(s)^{r-1}\right) .
$$

Proof. By (4.9), if (4.10) holds, 1 is not an eigenvalue of $\partial_{u} F\left(u_{0}, \lambda_{0}\right)$, and so $I d-$ $\partial_{u} F\left(u_{0}, \lambda_{0}\right)$ is a topological isomorphism. Hence we can apply Proposition 20.6 of [3] and conclude the first paragraph.

Again by (4.9), if (4.11) holds, 1 is an eigenvalue with positive eigenfunction of $\partial_{u} F\left(u_{0}, \lambda_{0}\right)$, so we can apply Propositions 20.7 and 20.8 of [3].

Finally, to prove (4.13), observe that from Proposition 20.8 of [3] it follows that

$$
\operatorname{sgn} \lambda^{\prime}(s)=\operatorname{sgn}(1-r(u(s), \lambda(s))) .
$$

Taking into account (4.9) it is not hard to show that

$$
\operatorname{sgn}(1-r(u(s), \lambda(s)))=\operatorname{sgn} \lambda_{1}\left(-\Delta-\lambda(s)+p u(s)^{p-1}, N-r u(s)^{r-1}\right) .
$$

This completes the proof.

We now analyze the behavior of the branch of solutions near a point $\left(\lambda_{0}, u_{0}\right)$ such that (4.11) holds. The fact that $\lambda^{\prime}(0)=0$ shows that this is actually a turning point of the branch of positive solutions (cf. Corollary 4.9 below). We are elucidating in what follows the direction of the turning. The essential ingredient is a Picone's type identity (see Section 4 in [8] and Lemma 4.1 in [30], for instance). Let $u, v \in C^{2}(\Omega) \cap C^{1}(\bar{\Omega})$ be such that $v / u \in C(\bar{\Omega})$ and $\Upsilon:[0, \infty) \mapsto \mathbb{R}$ an arbitrary $C^{1}$ function. Then

$$
\int_{\Omega} \Upsilon\left(\frac{v}{u}\right)(-v \Delta u+u \Delta v)=-\int_{\Omega} \Upsilon^{\prime}\left(\frac{v}{u}\right) u^{2} \nabla\left|\left(\frac{v}{u}\right)\right|^{2}-\int_{\partial \Omega} \Upsilon\left(\frac{v}{u}\right)\left[v \frac{\partial u}{\partial \nu}-u \frac{\partial v}{\partial \nu}\right] .
$$

Then we have the following important result.

Proposition 4.8. Assume $p \leq r$. Let $\left(\lambda_{0}, u_{0}\right)$ be a positive solution to (1.1) with $\lambda=\lambda_{0}$, such that (4.11) holds. Then $\lambda^{\prime \prime}(0)<0$, where $\lambda^{\prime \prime}(0)$ is defined in (4.12).

Proof. To determine the sign of $\lambda^{\prime \prime}(0)$, we use the Picone's identity (4.14) with $\Upsilon(t)=t^{2}$, $v=\Phi_{0}$ and $u=u_{0}$, to obtain

$$
(p-1) \int_{\Omega} u_{0}^{p-2} \Phi_{0}^{3}<(r-1) \int_{\partial \Omega} u_{0}^{r-2} \Phi_{0}^{3} .
$$

From (4.15) and as $p \leq r$ we can infer that $\lambda^{\prime \prime}(0)<0$. This concludes the proof. 
As an easy consequence of Lemma 4.7 (in particular relations (4.12) and (4.13)) and Proposition 4.8, we obtain:

Corollary 4.9. Let $\left(\lambda_{0}, u_{0}\right)$ be a positive solution to (1.1) with $\lambda=\lambda_{0}>0$, such that $\lambda_{1}\left(-\Delta-\lambda_{0}+p u_{0}^{p-1}, N-r u_{0}^{r-1}\right)=0$. Then, there exists $\varepsilon>0$ such that for each $\lambda \in\left(\lambda_{0}-\varepsilon, \lambda_{0}\right)$, (1.1) has two positive solutions, one of them stable and the other one unstable. Moreover, there exists a neighborhood $\mathcal{N}$ of $\left(\lambda_{0}, u_{0}\right)$ in $\mathbb{R} \times P$ such that (1.1) does not have positive solutions in $\mathcal{N}$ for $\lambda>\lambda_{0}$.

We are finally ready to prove the uniqueness of the stable solution.

Theorem 4.10. Assume that $p \leq r$. Then, the minimal solution is the unique positive stable solution to (1.1) for all $\lambda \in\left(0, \Lambda_{0}\right)$.

Proof. We first show that the minimal solution $u_{\lambda}$ is stable for all $\lambda \in\left(0, \Lambda_{0}\right)$. It is well known (see Proposition 20.4 in [3]) that the minimal solution is weakly stable, i.e.,

$$
\lambda_{1}\left(-\Delta-\lambda+p u_{\lambda}^{p-1}, N-r u_{\lambda}^{r-1}\right) \geq 0 \quad \text { for all } \lambda \in\left(0, \Lambda_{0}\right) .
$$

On the other hand, in a neighborhood $\mathcal{N}$ of $(\lambda, u)=(0,0)$, there exists a unique positive solution for fixed $\lambda$. Since the minimal solution exists for all $\lambda \in\left(0, \Lambda_{0}\right)$, the unique solution coincides with the minimal, so by Corollary 4.9 there exists $\underline{\lambda}$ such that for all $0<\lambda \leq \underline{\lambda}$ we have that

$$
\lambda_{1}\left(-\Delta-\lambda+p u_{\lambda}^{p-1}, N-r u_{\lambda}^{r-1}\right)>0 .
$$

Now, we can produce this branch to the right to reach a value $\lambda_{0} \leq \Lambda_{0}$ such that $\lambda_{1}(-\Delta-$ $\left.\lambda+p u_{\lambda}^{p-1}, N-r u_{\lambda}^{r-1}\right)>0$ for all $\lambda<\lambda_{0}$ and

$$
\lambda_{1}\left(-\Delta-\lambda_{0}+p u_{\lambda_{0}}^{p-1}, N-r u_{\lambda_{0}}^{r-1}\right)=0 .
$$

If $\lambda_{0}=\Lambda_{0}$ we have proved that the minimal solution is stable for all $\lambda<\Lambda_{0}$. So assume that $\lambda_{0}<\Lambda_{0}$. Thanks to (4.16) and Corollary 4.9, there exists a value $\lambda_{1} \in\left(\lambda_{0}, \Lambda_{0}\right)$ such that

$$
\lambda_{1}\left(-\Delta-\lambda_{1}+p u_{\lambda_{1}}^{p-1}, N-r u_{\lambda_{1}}^{r-1}\right)>0,
$$

and by Lemma 4.7 , part 1 , we can continue the branch to the left of $\lambda_{1}$. Denote

$$
\Gamma=\left\{(\lambda, u(\lambda)): \lambda \leq \lambda_{1}\right\}
$$

Now two possibilities may arise:

1. There exists $\lambda_{2}<\lambda_{1}$ such that $\lambda_{1}\left(-\Delta-\lambda_{2}+p u\left(\lambda_{2}\right)^{p-1}, N-r u\left(\lambda_{2}\right)^{r-1}\right)=0$.

2. The branch $\Gamma$ can be continued for all $\lambda \leq \lambda_{1}$ with $\lambda_{1}\left(-\Delta-\lambda+p u(\lambda)^{p-1}, N-\right.$ $\left.r u(\lambda)^{r-1}\right)>0$.

If the first possibility holds, then Corollary 4.9 is contradicted. In the second possibility, $\Gamma$ does not reach negative values of $\lambda$ by Lemma 4.1. So, again two situations are possible:

1. The branch $\Gamma$ meets the real axis $\{(\lambda, 0)\}$. 
2. The branch $\Gamma$ reaches the minimal solution at some point $\left(\lambda_{3}, u_{\lambda_{3}}\right)$.

If $\Gamma$ meets the axis $\{(\lambda, 0)\}$, since we know that the unique bifurcation point from the trivial solution is $\lambda=0$, then $\Gamma$ reaches at $(0,0)$. But, as remarked before, in a neighborhood $\mathcal{N}$ of $(\lambda, u)=(0,0)$ there exists a unique solution, in fact the minimal solution. So, the second possibility occurs. If $\lambda_{3}$ is such that $u_{\lambda_{3}}$ satisfies (4.17), Corollary 4.9 leads to a contradiction. However, if $\lambda_{3}$ is such that $u_{\lambda_{3}}$ satisfies $\lambda_{1}\left(-\Delta-\lambda_{3}+p u_{\lambda_{3}}^{p-1}, N-\right.$ $\left.r u_{\lambda_{3}}^{r-1}\right)>0$, we know that in a neighborhood $\mathcal{M}$ of $\left(\lambda_{3}, u_{\lambda_{3}}\right)$ there exists a unique solution, a contradiction. This contradiction shows that the minimal solution $u_{\lambda}$ is stable for all $\lambda \in\left(0, \Lambda_{0}\right)$ and neutrally stable for $\lambda=\Lambda_{0}$.

Now, assume that for some $\lambda_{0} \in\left(0, \Lambda_{0}\right)$ there exists a second stable solution $v_{0}>u_{\lambda_{0}}$. We argue as in the first part of the proof. By Lemma 4.7, part 1, there exists a branch, say $\Gamma^{\prime}$, of stable solutions of the form $(\lambda(s), v(s)), s \in I$, with $\lambda(0)=\lambda_{0}, v(0)=v_{0}$. Moreover, we can continue this branch to the left until there exists a value $\lambda^{*}$ in which it is noncontinuable. Since, by Lemma 4.1, part 3, all solutions are unstable for $\lambda \leq 0$, it follows that $\lambda^{*} \geq 0$.

If $\lambda^{*}>0$, we would have thanks to Lemma 4.7, part 1 , that $\lambda_{1}\left(-\Delta-\lambda^{*}+p v_{\lambda^{*}}^{p-1}, N-\right.$ $\left.r v_{\lambda^{*}}^{r-1}\right)=0$, and we arrive at a contradiction with Corollary 4.9. Hence $\lambda^{*}=0$. Moreover, the branch $\Gamma^{\prime}$ has to degenerate at $(0,0)$, otherwise we could continue it thanks to Lemma 4.7, part 1 . However, this contradicts the uniqueness of solutions for $\lambda \sim 0$, and the uniqueness of the stable solution is proved.

\section{Behavior of solutions for large $|\lambda|$}

This section is devoted to obtain the behavior of all positive solutions to (1.1) when $\lambda \nearrow \infty$ or $\lambda \searrow-\infty$. All the proofs are based on the well-known blow-up argument of Gidas and Spruck, [23]. An essential role in them is played by a nonexistence result for problems with nonlinear boundary conditions in a half-space obtained in [27].

We begin by considering the behavior of the positive solutions for $\lambda \rightarrow+\infty$ in the case $p<1 \leq r$, assuming that $r$ is subcritical.

Theorem 5.1. Assume that $0<p<1 \leq r<d /(d-2)$. For every $\lambda_{0}>0$, there exist positive constants $C_{1}, C_{2}$ such that, for every nonnegative solution u to (1.1) with $\lambda \geq \lambda_{0}$, we have

$$
C_{1} \lambda^{-\frac{1}{1-p}} \leq \max _{\Omega} u \leq C_{2} \lambda^{-\frac{1}{1-p}}
$$

Proof. We are using as in [21] a blow-up argument. Since this argument will also be used in the next theorems, we detail it in this case. Assume that the right-hand side inequality in (5.1) does not hold. Then there exist sequences $\lambda_{n} \nearrow \infty$, and $u_{n} \in C^{2, \alpha}(\bar{\Omega})$ solutions to (1.1) with $\lambda=\lambda_{n}$ such that

$$
\lambda_{n}^{\frac{1}{1-p}} M_{n} \nearrow \infty
$$

where $M_{n}$ stands for the maximum of $u_{n}$. Take a point $x_{n} \in \bar{\Omega}$ where $u_{n}$ attains its maximum and assume with no loss of generality that $x_{n} \rightarrow x_{0} \in \bar{\Omega}$. We need to distinguish two cases: $x_{0} \in \Omega$ or $x_{0} \in \partial \Omega$.

Case 1. $x_{0} \in \Omega$. Introduce the scaled functions

$$
v_{n}(y)=\frac{u_{n}\left(x_{n}+\lambda_{n}^{-1 / 2} y\right)}{M_{n}},
$$


which verify $v_{n}(0)=1,0 \leq v_{n} \leq 1$ and

$$
-\Delta v_{n}=v_{n}-\frac{1}{\lambda_{n} M_{n}^{1-p}} v_{n}^{p} \quad \text { in } \Omega_{n}
$$

where $\Omega_{n}=\lambda_{n}^{1 / 2}\left(-x_{n}+\Omega\right)$. It is easily seen that $\Omega_{n} \rightarrow \mathbb{R}^{d}$ as $n \rightarrow \infty$.

Since $v_{n}$ is bounded, it is standard to obtain bounds in $C_{\text {loc }}^{1, \alpha}\left(\mathbb{R}^{d}\right)$, which then provide with bounds in $C_{\text {loc }}^{2, \alpha}\left(\mathbb{R}^{d}\right)([24])$. Then, passing to a subsequence, we have that $v_{n} \rightarrow v$ in $C_{\text {loc }}^{2}\left(\mathbb{R}^{d}\right)$, where $v$ is a solution to

$$
-\Delta v=v \quad \text { in } \mathbb{R}^{d}
$$

with $0 \leq v \leq 1, v(0)=1$. We claim that this is impossible. Indeed, let $\lambda_{1}(R)$ be the principal eigenvalue of $-\Delta$ under homogeneous Dirichlet boundary conditions in the ball $B_{R}$ of radius $R$ centered at the origin, with associated positive eigenfunction $\phi_{R}$. If we multiply (5.3) by $\phi_{R}$ and integrate in $B_{R}$, we have

$$
\left(\lambda_{1}(R)-1\right) \int_{B_{R}} v \phi_{R} \geq 0
$$

since $\partial \phi_{R} / \partial \nu<0$ on $\partial B_{R}$. Taking into account that $\lambda_{1}(R) \rightarrow 0$ as $R \rightarrow \infty$, we arrive at $v=0$ in $B_{R}$ for large $R$, which is impossible, as $v(0)=1$. Hence $M_{n} \lambda_{n}^{1 /(1-p)} \rightarrow \infty$ is impossible, and the right-hand inequality of (5.1) is proved in this case.

Case 2. $x_{0} \in \partial \Omega$. As usual, before introducing the scaling, we need to straighten the boundary of $\Omega$ near $x_{0}$. Without loss of generality, we may assume that $x_{0}=0$, and that $\nu\left(x_{0}\right)=-e_{d}$, the last vector of the canonical basis of $\mathbb{R}^{d}$. Since $\Omega$ is $C^{2, \gamma}$, there exist $R>0$ and $\varphi \in C^{2, \gamma}\left(B(0, R) \cap\left\{x_{d}=0\right\}\right)$ verifying $\varphi(0)=0, \nabla \varphi(0)=0$ and writing $x=\left(x^{\prime}, x_{d}\right)$, we have $\Omega \cap B(0, R)=\left\{x: x_{d}>\varphi\left(x^{\prime}\right)\right\}$ and $\partial \Omega \cap B(0, R)=\left\{x: x_{d}=\varphi\left(x^{\prime}\right)\right\}$. Then the diffeomorphism $y=h(x)$ given by $y^{\prime}=x^{\prime}, y_{d}=x_{d}-\varphi\left(x_{d}\right)$ maps $B(0, R)$ onto a neighborhood $V$ of $y=0$ in $\mathbb{R}^{d}$, while it maps $\Omega \cap B(0, R)$ onto $V^{+}=V \cap \mathbb{R}_{+}^{d}$ and $\partial \Omega \cap B(0, R)$ onto $V \cap \partial \mathbb{R}_{+}^{d}$. Then problem (1.1) gets transformed into:

$$
\left\{\begin{array}{lc}
-\Delta u+\sum_{i=1}^{d-1} a_{i}(y) u_{y_{i} y_{d}}-\left|\nabla \varphi\left(y^{\prime}\right)\right|^{2} u_{y_{d} y_{d}}+b(y) u_{y_{d}}=\lambda u-u^{p} & y \in V^{+}, \\
\nabla u \cdot \nu_{1}(y)=u^{r} & y \in V \cap \partial \mathbb{R}_{+}^{d},
\end{array}\right.
$$

where

$$
a_{i}=2 \varphi_{x_{i}}, \quad b(y)=\Delta \varphi, \quad \nu_{1}=\left(\nu^{\prime},-\nu^{\prime} \nabla \varphi+\nu_{d}\right),
$$

and all functions are evaluated at $x=h^{-1}(y)$.

At this point, we claim that $\lambda_{n} M_{n}^{-2(r-1)} \rightarrow \infty$. Since this is clear in the particular case $r=1$, we may assume for the moment $r>1$. If we had $\lambda_{n} M_{n}^{-2(r-1)}$ bounded for some subsequence, we can assume $\lambda_{n} M_{n}^{-2(r-1)} \rightarrow c$, with $c \geq 0$. Let $y_{n}=h\left(x_{n}\right)$, and introduce the functions

$$
v_{n}(y)=\frac{u_{n}\left(y_{n}+M_{n}^{-(r-1)} y\right)}{M_{n}}
$$


(observe that we must have in this case $M_{n} \rightarrow \infty$ ). Then $v_{n}$ are solutions to

$$
\left\{\begin{array}{c}
-\Delta v+\sum_{i=1}^{d-1} a_{i, n}(y) v_{y_{i} y_{d}}-a_{d, n}(y) v_{y_{d} y_{d}}+M_{n}^{-(r-1)} b_{n}(y) v_{y_{d}} \\
=\frac{\lambda_{n}}{M_{n}^{2(r-1)} v-\frac{1}{M_{n}^{2(r-1)+1-p}} v^{p}} \quad y \in U_{n}, \\
\nabla v \cdot \nu_{1, n}(y)=v^{r} \quad y \in \partial U_{n} \cap \mathbb{R}_{+}^{d,},
\end{array}\right.
$$

where $U_{n}:=M_{n}^{r-1}\left(B(0, R) \cap \mathbb{R}_{+}^{d}\right)-y_{n}, a_{i, n}=a_{i}\left(y_{n}+M_{n}^{-(r-1)} y\right), 1 \leq i \leq d-1$, $a_{d, n}(y)=\left|\nabla \varphi\left(y_{n}^{\prime}+M_{n}^{-(r-1)} y^{\prime}\right)\right|^{2}, b_{n}(y)=b\left(y_{n}+M_{n}^{-(r-1)} y\right), \nu_{1, n}(y)=\nu_{1}\left(y_{n}+M_{n}^{-(r-1)} y\right)$.

We need to distinguish two subcases:

(a) $\lambda_{n}^{1 / 2} \operatorname{dist}\left(x_{n}, \partial \Omega\right)$ is unbounded. Passing to a subsequence, we may assume that $\lambda_{n}^{1 / 2} \operatorname{dist}\left(x_{n}, \partial \Omega\right) \rightarrow+\infty$. In this case $U_{n} \rightarrow \mathbb{R}_{+}^{d}$, and the contradiction is reached as in Case 1, the limit problem being (5.3).

(b) $\lambda_{n}^{1 / 2} \operatorname{dist}\left(x_{n}, \partial \Omega\right)$ is bounded. Passing to a subsequence, $\lambda_{n}^{1 / 2} \operatorname{dist}\left(x_{n}, \partial \Omega\right) \rightarrow \gamma \geq 0$, so that $U_{n} \rightarrow\left\{y_{d} \geq-\gamma\right\}$. Since $0 \leq v_{n} \leq 1$, we can obtain $C_{\text {loc }}^{2, \alpha}\left(\overline{\mathbb{R}_{+}^{d}}\right)$ bounds (cf. [28]), and then pass to the limit through a subsequence to obtain that $v_{n} \rightarrow v$, which is a solution to

$$
\left\{\begin{aligned}
-\Delta v & =c v & & \text { in }\left\{y_{d}>-\gamma\right\} \\
\frac{\partial v}{\partial \nu} & =v^{r} & & \text { on } y_{d}=-\gamma,
\end{aligned}\right.
$$

which in addition verifies $0 \leq v \leq 1, v(0)=1$. With a further translation (which does not change the equation) we may assume problem (5.4) is posed in $\mathbb{R}_{+}^{d}$.

If $c=0$, this contradicts Theorem 1.2 in [27]. If $c \neq 0$, we can multiply (5.4) by the eigenfunction $\phi_{R}$ associated to the first eigenvalue of $-\Delta$ under homogeneous Dirichlet boundary conditions in a ball of radius $R$ contained in $\mathbb{R}_{+}^{d}$ to obtain as before that $\lambda_{1}(R) \geq$ $c$, which is clearly impossible for large $R$. Hence we arrive at a contradiction which shows the claim.

We now introduce the functions

$$
w_{n}(y)=\frac{u_{n}\left(y_{n}+\lambda_{n}^{-1 / 2} y\right)}{M_{n}},
$$

which are solutions to

$$
\left\{\begin{array}{cc}
-\Delta w+\sum_{i=1}^{d-1} a_{i, n}(y) w_{y_{i} y_{d}}-a_{d, n}(y) w_{y_{d} y_{d}}+\lambda_{n}^{-1 / 2} b_{n}(y) w_{y_{d}} & \\
=w-\frac{1}{\lambda_{n} M_{n}^{1-p}} w^{p} & y \in U_{n}, \\
\nabla w \cdot \nu_{1, n}(y)=M_{n}^{r-1} \lambda_{n}^{-1 / 2} w^{r} & y \in \partial U_{n} \cap \partial \mathbb{R}_{+}^{d},
\end{array}\right.
$$

where now $U_{n}:=\lambda_{n}^{1 / 2}\left(B(0, R) \cap \mathbb{R}_{+}^{d}\right), a_{i, n}=a_{i}\left(y_{n}+\lambda_{n}^{-1 / 2} y\right), 1 \leq i \leq d-1, a_{d, n}(y)=$ $\left|\nabla \varphi\left(y_{n}^{\prime}+\lambda_{n}^{-1 / 2} y^{\prime}\right)\right|^{2}, b_{n}(y)=b\left(y_{n}+\lambda_{n}^{-1 / 2} y\right), \nu_{1, n}(y)=\nu_{1}\left(y_{n}+\lambda_{n}^{-1 / 2} y\right)$. Passing to the limit as before we arrive at $w_{n} \rightarrow w$, which either solves

$$
-\Delta w=w \quad \text { in } \mathbb{R}_{+}^{d},
$$


or

$$
\left\{\begin{aligned}
-\Delta w & =w & & \text { in } \mathbb{R}_{+}^{d}, \\
\frac{\partial w}{\partial \nu} & =0 & & \text { on } \partial \mathbb{R}_{+}^{d} .
\end{aligned}\right.
$$

Extending $w$ to all of $\mathbb{R}^{d}$ as an even function in the second case, we obtain a nonnegative nontrivial solution to $-\Delta w=w$ in $\mathbb{R}^{d}$ in both cases, which has been shown to be impossible, and hence the right-hand side of (5.1) is proved.

Since the arguments used to prove the left-hand inequality in (5.1) are similar, we are only sketching the proof. Thus assume $M_{n} \lambda_{n}^{1 /(1-p)} \rightarrow 0$ for a certain sequence $\lambda_{n} \nearrow \infty$ with corresponding solutions $u_{n}$. In Case 1 , we set

$$
v_{n}(y)=\frac{u_{n}\left(x_{n}+M_{n}^{\frac{1-p}{2}} y\right)}{M_{n}},
$$

and passing to the limit we arrive at a solution $v$ to

$$
-\Delta v=-v^{p} \quad \text { in } \mathbb{R}^{d},
$$

with $0 \leq v \leq 1, v(0)=1$, which is clearly impossible, since $v$ attains its maximum at zero, and then $-\Delta v(0) \geq 0$.

In Case 2, we set

$$
v_{n}(y)=\frac{u_{n}\left(y_{n}+M_{n}^{\frac{1-p}{2}} y\right)}{M_{n}},
$$

and in the limit obtain equation (5.5) or

$$
\left\{\begin{aligned}
-\Delta v & =-v^{p} & \text { in } & \mathbb{R}_{+}^{d}, \\
\frac{\partial v}{\partial \nu} & =0 & \text { on } & \partial \mathbb{R}_{+}^{d} .
\end{aligned}\right.
$$

In the second case, the function $v$ can then be extended as an even function to all of $\mathbb{R}^{d}$, verifying (5.5), which as we have seen is impossible. This completes the proof of (5.1).

A similar proof as that of the upper estimate in (5.1) can be made for $p>1$ in the cases where positive solutions exist for large $\lambda$, that is $r \leq 1$ or $r>1$ and $p>2 r-1$ (we recall that no solutions exist for large $\lambda$ if $p=1)$. This, together with $(2.3)$, leads to:

Theorem 5.2. Assume that $p>1$ and $r \leq 1$ or $1<r<d /(d-2)$ and $p>2 r-1$. For every $\lambda_{0}>0$, there exists a positive constant $C$ such that, for every nonnegative solution $u$ to (1.1) with $\lambda \geq \lambda_{0}$, we have

$$
\lambda^{-\frac{1}{1-p}} \leq \max _{\Omega} u \leq C \lambda^{-\frac{1}{1-p}} .
$$

Proof. The lower inequality in (5.6) is (2.3) in Lemma 2.3. To prove the upper inequality, assume it does not hold, that is, there exist sequences $\lambda_{n} \nearrow \infty$ and solutions $u_{n}$ verifying (5.2). It is easily seen that the proof is identical to that of Theorem 5.1 provided we show that $\lambda_{n} M_{n}^{-2(r-1)} \nearrow \infty$. We introduce the functions

$$
v_{n}(y)=\frac{u_{n}\left(y_{n}+M_{n}^{\frac{1-p}{2}} y\right)}{M_{n}},
$$


and passing to the limit obtain a positive solution to the equation

$$
\left\{\begin{aligned}
-\Delta v & =-v^{p} & & \text { in } \mathbb{R}_{+}^{d}, \\
\frac{\partial v}{\partial \nu} & =0 & & \text { on } \partial \mathbb{R}_{+}^{d},
\end{aligned}\right.
$$

which has been shown to be impossible. We remark that in this case the left inequality in (5.6) implies $-\Delta u \leq 0$, and thus $x_{n} \in \partial \Omega$ for all $n$.

We now turn to consider the cases where positive solutions exist for large negative $\lambda$, namely $r<1, p \geq 1$ and $1<r<d /(d-2), p<2 r-1$. We collect them both in a single statement, and only sketch its proof, since it is entirely similar to that of Theorem 5.1.

Theorem 5.3. Assume that $0<r<1 \leq p$ or $1<r<d /(d-2)$ and $p<2 r-1$. For every $\lambda_{0}<0$, there exist positive constants $C_{1}, C_{2}$ such that, for every nonnegative solution $u$ to (1.1) with $\lambda \leq \lambda_{0}$, we have

$$
C_{1}(-\lambda)^{\frac{1}{2(r-1)}} \leq \max _{\Omega} u \leq C_{2}(-\lambda)^{\frac{1}{2(r-1)}} .
$$

Proof. We begin with the case $0<r<1 \leq p$. Thus assume that there exist sequences $\lambda_{n} \searrow-\infty$ and corresponding solutions $u_{n} \in C^{2, \alpha}(\bar{\Omega})$ such that

$$
M_{n}\left(-\lambda_{n}\right)^{-1 /(2(r-1))} \nearrow \infty,
$$

where $M_{n}$ stands for the maximum of $u_{n}$. Take a point $x_{n} \in \partial \Omega$ where $u_{n}$ attains its maximum and assume $x_{n} \rightarrow x_{0} \in \partial \Omega$ (observe that $\lambda<0$ implies that the maximum of a solution is attained at $\partial \Omega)$.

We claim that $M_{n}^{p-1}\left(-\lambda_{n}\right)^{-1}$ is bounded. Since this is immediate if $p=1$, we restrict to $p>1$ and assume that, passing to a subsequence,

$$
M_{n}^{p-1}\left(-\lambda_{n}\right)^{-1} \nearrow+\infty
$$

and in particular $M_{n} \nearrow \infty$. We introduce the scaled functions

$$
w_{n}(y)=\frac{u_{n}\left(x_{n}+M_{n}^{(1-p) / 2} y\right)}{M_{n}},
$$

and pass to the limit to obtain a positive solution $w$ to

$$
\left\{\begin{aligned}
-\Delta w & =-w^{p} & & \text { in } \mathbb{R}_{+}^{d}, \\
\frac{\partial w}{\partial \nu} & =0 & & \text { on } \partial \mathbb{R}_{+}^{d},
\end{aligned}\right.
$$

which is impossible. This proves the claim. Thus, we may assume that there exists $c \geq 0$ such that $M_{n}^{p-1}\left(-\lambda_{n}\right)^{-1} \rightarrow c$.

Defining the functions

$$
v_{n}(y)=\frac{u_{n}\left(x_{n}+\left(-\lambda_{n}\right)^{-1 / 2} y\right)}{M_{n}}
$$


and passing to the limit in a similar way, we obtain a positive solution $v$ to

$$
\left\{\begin{aligned}
-\Delta v & =-v-c v^{p} & & \text { in } \mathbb{R}_{+}^{d}, \\
\frac{\partial v}{\partial \nu} & =0 & & \text { on } \partial \mathbb{R}_{+}^{d},
\end{aligned}\right.
$$

which is again a contradiction.

To prove the lower inequality in (5.7), we assume that for a sequence $\lambda_{n} \searrow-\infty$, with corresponding solutions $u_{n}$, we have

$$
M_{n}\left(-\lambda_{n}\right)^{-\frac{1}{2(r-1)}} \rightarrow 0
$$

where $M_{n}=\max _{\bar{\Omega}} u_{n}$. We remark that, thanks to the first part of the proof, we have $M_{n} \rightarrow 0$. With the rescaled functions

$$
z_{n}(y)=\frac{u_{n}\left(x_{n}+M_{n}^{1-r} y\right)}{M_{n}},
$$

and passing to the limit, we obtain a solution to

$$
\left\{\begin{aligned}
-\Delta w & =0 & & \text { in } \mathbb{R}_{+}^{d}, \\
\frac{\partial w}{\partial \nu} & =w^{r} & & \text { on } \partial \mathbb{R}_{+}^{d},
\end{aligned}\right.
$$

with $0<w \leq 1$ and $w(0)=1$. However, Theorem 1.2 in [27] cannot be applied in this setting, since it is required there that $r \geq 1$. Thus we are including an alternative proof, along the lines of Theorem 1 (iii) in [21].

Let $D \subset \mathbb{R}_{+}^{d}$ be a smooth bounded domain such that $\Gamma:=\partial D \cap \partial \mathbb{R}_{+}^{d}=\overline{B(0,1)} \cap \partial \mathbb{R}_{+}^{d}$, and set $\Gamma^{\prime}=\partial D \cap \mathbb{R}_{+}^{d}$. Define $D_{n}=n D, \Gamma_{n}=n \Gamma, \Gamma_{n}^{\prime}=n \Gamma^{\prime}$, for $n \in \mathbb{N}$. According to Theorem 8 in [21], the eigenvalue problem

$$
\begin{cases}\Delta z=0 & \text { in } D_{n} \\ \frac{\partial z}{\partial \nu}=\sigma z & \text { on } \Gamma_{n} \\ z=0 & \text { on } \Gamma_{n}^{\prime}\end{cases}
$$

admits a first eigenvalue $\sigma=\sigma_{1, n}$ with a positive associated eigenfunction $\varphi_{n} \in H^{1}\left(D_{n}\right) \cap$ $W^{2, q}\left(D_{n}\right) \cap C^{2, \gamma}\left(D_{n} \cup T\right)$, for all $1<q<4 / 3$ and any closed $T \subset B(0, n) \cap \partial \mathbb{R}_{+}^{d}$. In addition, $\sigma_{1, n} \rightarrow 0$.

If we multiply the equation in (5.8) by $\varphi_{n}$ and integrate

$$
0=\int_{\Gamma_{n}} w^{r} \varphi_{n}-\sigma_{n} \int_{\Gamma_{n}} w \varphi_{n}-\int_{\Gamma_{n}^{\prime}} w \frac{\partial \varphi_{n}}{\partial \nu} \geq\left(1-\sigma_{n}\right) \int_{\Gamma_{n}} w \varphi_{n},
$$

since $\partial \varphi_{n} / \partial \nu<0$ on $\Gamma_{n}^{\prime}$ and $w^{r} \geq w$ (this follows because $r \leq 1$ and $0 \leq w \leq 1$ ), which is not possible provided $n$ is large.

The proof of the remaining case $r>1$ and $p<2 r-1$ is entirely similar (actually, the scaled functions are constructed symmetrically since now $r>1$ ) and we leave the proof to the reader.

Acknowledgements. Supported by MEC and FEDER under grants MTM2005-06480 (JGM \& JDR), BFM2003-06446 and MTM2006-07932 (AS), and ANPCyT PICT No. 03-05009 (JDR). JDR is a member of CONICET. 


\section{References}

[1] S. Agmon, A. Douglis And L. Nirenberg, Estimates near the boundary for solutions of elliptic partial differential equations satisfying general boundary conditions I, Comm. Pure Appl. Math. 12 (1959), 623-727.

[2] H. Amann, Nonlinear elliptic equations with nonlinear boundary conditions, In "New Developments in differential equations" (W. Eckhaus, ed.), Math Studies 21, NorthHolland, Amsterdam (1976), 43-63.

[3] H. Amann, Fixed points equations and nonlinear eigenvalue problems in ordered Banach spaces, SIAM Review 18 (1976), 620-709.

[4] H. Amann And J. LóPEz-Gómez, A priori bounds and multiple solutions for superlinear indefinite elliptic problems, J. Differential Equations 146 (1998), 336-374.

[5] A. Ambrosetti And P.H. Rabinowitz, Dual variational methods in critical point theory and applications, J. Funct. Anal. 14 (1973), 349-381.

[6] F. Andreu, J. M. Mazón, J. Toledo And J. D. Rossi, Porous media equation with absorption and a nonlinear boundary condition, Nonlinear Anal. 49 (2002), 541563.

[7] J. M. Arrieta, R. Pardo and A. Rodríguez-Bernal, Bifurcation and stability of equilibria with asymptotically linear boundary conditions at infinity, to appear in Proc. Royal Soc. Edinburgh A.

[8] H. Berestycki, I. Capuzzo-Dolcetta and L. Nirenberg, Variational methods for indefinite superlinear homogeneous elliptic problems, NoDEA Nonlinear Diff. Eqns. Appl. 2 (1995), 553-572.

[9] H. Brezis And L. Oswald, Remarks on sublinear elliptic equations, Nonlinear Anal. 10 (1986), 55-64.

[10] S. Cano-Casanova, On the positive solutions of the logistic weighted ellipitc BVP with sublinear mixed boundary conditions, in "Spectral Theory and Nonlinear Analysis with Applications to Spatial Ecology" (S. Cano-Casanova, J. Lopez-Gomez, C. MoraCorral, eds), World Scientific, (2005), 1-15.

[11] S. Cano-Casanova And J. López-Gómez, Properties of the principal eigenvalues of a general class of non-classical mixed boundary value problems, J. Differential Equations 178 (2002), 123-211.

[12] R. S. Cantrell And C. Cosner, On the effects of nonlinear boundary conditions in diffusive logistic equations on bounded domains, J. Differential Equations 231 (2006), 768-804.

[13] P. Cherrier, Problèmes de Neumann non linéaires sur les variétés Riemanniennes, J. Funct. Anal. 57 (1984), 154-206.

[14] M. Chipot, M. Fila And P. QuitTner, Stationary solutions, blow up and convergence to stationary solutions for a semilinear parabolic equation with nonlinear boundary conditions, Acta Math. Univ. Comenianae 60 (1991), 35-103. 
[15] M. Chipot And P. QuitTner, Equilibria, connecting orbits and a priori bounds for semilinear parabolic equations with nonlinear boundary conditions, J. Dynamics Diff. Equations 16 (2004), 91-138.

[16] M. Del Pino And C. Flores, Asymptotic behavior of best constants and extremals for trace embeddings in expanding domains, Comm. Partial Differential Equations 26 (2001), 2189-2210.

[17] M. Fila, J. J. L. Velázquez And M. Winkler, Blow-up on the boundary for a semilinear parabolic problem, In Progress in Nonlinear Differential Equations and Their Applications, Vol. 64, Birkhäuser, Basel, 2005, 137-150.

[18] J. M. Fraile, P. Koch Medina, J. López-Gómez and S. Merino, Elliptic eigenvalue problems and unbounded continua of positive solutions of a semilinear elliptic equation, J. Differential Equations 127 (1996), 295-319.

[19] J. L. GÁmez, Sub- and super-solutions in bifurcation problems, Nonlinear Anal. 28 (1997), 625-632.

[20] J. García-Melián, J. D. Rossi and J. Sabina DE Lis, A bifurcation problem governed by the boundary condition I, to appear in NoDEA Nonlinear Diff. Eqns. Appl.

[21] J. García-Melián, J. D. Rossi and J. Sabina De Lis, A bifurcation problem governed by the boundary condition II, Proc. London Math. Soc. 94 (2007), 1-25.

[22] J. García-Melián, J. D. Rossi and J. Sabina de Lis, Existence and uniqueness of positive solutions to elliptic problems with sublinear mixed boundary conditions, preprint.

[23] B. Gidas And J. Spruck, A priori bounds for positive solutions of nonlinear elliptic equations, Comm. Partial Differential Equations 6 (1981), 883-901.

[24] D. Gilbarg And N. S. Trudinger, Elliptic partial differential equations of second order, Springer Verlag (1983).

[25] R. Gómez-REÑAsco AND J. LÓPEZ-Gómez, The effect of varying coefficients on the dynamics of a class of superlinear indefinite reaction-diffusion equations, J. Differential Equations 167 (2000), 36-72.

[26] P. Hess, On the uniqueness of positive solutions of nonlinear elliptic boundary value problems, Math. Z. 154 (1977), 17-18.

[27] B. Hu, Nonexistence of a positive solution of the Laplace equation with a nonlinear boundary condition, Differential Integral Equations 7 (1994), 301-313.

[28] O. A. Ladyzhenskaya and N. N. Uralt'Seva, Linear and quasilinear elliptic equations, Academic Press, New York (1968).

[29] A. W. Leung And Q. Zhang, Reaction diffusion equations with non-linear boundary conditions, blowup and steady states, Math. Meth. Appl. Sci. 21 (1998), 1593-1617. 
[30] J. LóPEz-Gómez, On the existence of positive solutions for some indefinite superlinear elliptic problems, Comm. Partial Differential Equations 22 (1997), 1787-1804.

[31] J. López-Gómez, V. Márquez and N. Wolanski, Dynamic behavior of positive solutions to reaction-diffusion problems with nonlinear absorption through the boundary, Rev. Un. Mat. Argentina 38 (1993), 196-209.

[32] C. Morales-Rodrigo And A. SuÁrez, Some elliptic problems with nonlinear boundary conditions, in "Spectral Theory and Nonlinear Analysis with Applications to Spatial Ecology" (S. Cano-Casanova, J. Lopez-Gomez, C. Mora-Corral, eds), World Scientific, (2005), 175-199.

[33] C. Morales-Rodrigo And A. SuÁrez, Uniqueness of solution for elliptic problems with nonlinear boundary conditions, Comm. Appl. Nonlinear Anal. 13 (2006), 69-78.

[34] C. V. PAO, Nonlinear parabolic and elliptic equations, Plenum, New York, (1992).

[35] P. QuitTner, On global existence and stationary solutions for two classes of semilinear parabolic problems, Comment. Math. Univ. Carolinae 34 (1993), 105-124.

[36] P. H. Rabinowitz, On bifurcation from infinity, J. Differential Equations 14 (1973), 462-475.

[37] A. Rodríguez-Bernal And A. TAjdine, Nonlinear balance for reaction-diffusion equations under nonlinear boundary conditions: dissipativity and blow-up, J. Differential Equations 169 (2001), 332-372.

[38] J. D. Rossi, Elliptic problems with nonlinear boundary conditions and the Sobolev trace theorem, Handbook of Differential Equations: Stationary partial differential Equations, Vol. 2, Chapter 5. Edited by M. Chipot and P. Quittner. Elsevier (2005), 311-406.

[39] J. SERRIN, Nonlinear Elliptic Equations of Second Order, AMS Symposium in Partial Differential Equations, Berkeley, August 1971.

[40] K. UMEZU, Bifurcation from infinity for asymptotically linear elliptic eigenvalue problems, J. Math. Anal. Appl. 267 (2002), 651-664.

[41] K. UMEZU, Behavior and stability of positive solutions of nonlinear elliptic boundary value problems arising in population dynamics, Nonlinear Anal. 49 (2002), 817-840.

[42] K. UMEZU, Local bifurcation analysis and stability of steady-state solutions for diffusive equations with nonlinear boundary conditions, Comm. Appl. Anal. 8 (2004), $533-547$.

[43] Y. WU AND M. WANG, Existence and nonexistence of global solution of nonlinear parabolic equation with nonlinear boundary condition, Chin. Ann. Math. 16B (1995), $371-378$. 